\title{
1 Genome-wide analysis of the cupin superfamily in cowpea (Vigna
}

2 unguiculata)

3 Antônio J. Rocha ${ }^{1^{*}}$, Mario Ramos de Oliveira Barsottini ${ }^{2}$ Ana Luiza Sobral

4 Paiva $^{1}$, José Hélio Costa ${ }^{1}$ Thalles Barbosa Grangeiro ${ }^{1}$

$6 \quad{ }^{1}$ Departamento de Bioquímica e Biologia Molecular, Centro de Ciências, Campus do

7 Pici, Universidade Federal do Ceará, Fortaleza, Ceará, 60.440-900, Brazil

$8 \quad{ }^{2}$ Laboratory of Genome e BioEnergy-LGE. Institute of Biology, State University of

9 Campinas, Campinas, São Paulo, Brazil

${ }^{3}$ Laboratório de Genética Molecular, Departamento de Biologia, Centro de Ciências,

*To whom all correspondence should be addressed

E-mail: antonionubis@gmail.com

\section{Abstract}

Cowpea [Vigna unguiculata (L.)Walp.] is an essential food crop that is cultivated in many important arid and semi-arid regions of the world. In this study the genome-wide database of cowpea genes was accessed in search of genomic sequences coding for globulins, specifically members of the cupin superfamily, a well-documented multigenic family belonging to the globulin protein class. A total of seventy-seven genes belonging to the cupin superfamily were found and divided into six families. We classify $V$. unguiculata genes into two subgroups: classical cupins with one cupin domain (fifty-nine proteins) and bicupins with two cupin domains (eighteen members). In addition, a search for cupin members in other closely related species of the fabaceae family [V. angularis, V. radiatam and Phaseolus vulgaris (common bean)] was performed. Based on those data, a detailed characterization and comparison of the cupin genes on these species was performed with the aim to better understand the connection and functions of cupin proteins from different, but related, plant species. This study was the first attempt to investigate the cupin superfamily in $V$. unguiculata, allowing the identification of six cupins families and better understand the structural features of those proteins, such as number of domains alternative splicing.

\section{Keywords:}

Vicilin, Leguminous, aminoacid sequences, protein domain, 


\section{Introduction}

Cowpea [Vigna unguiculata (L.)Walp.] is an important food crop that is cultivated in arid and semi-arid regions of Africa, Asia and Americas. In Brazil, it is mainly found in the northeast region, where it is a source of food for the population of that region (Ehlers et al, 1999).

Cowpea seed storage proteins are classified in four groups based on their solubility: albumins (water-soluble proteins), prolamins (alcohol-soluble), glutelins (acid or alkali-soluble) and globulins (diluted saline solution-soluble) (Osborne 1924). Globulins, in turn, are divided into two subgroups according to their sedimentation coefficients: 7S and $11 \mathrm{~S}$ globulin-types, respectively known as vicilins and legumins (Ponzoni et al, 2018). Vicilins constitute the major source of nutrients during cowpea seed development (Kriz et al, 1999) and are composed of several isoforms encoded by multigenic families which are categorized based on the occurrence or not of enzymatic activity (Shotwell and Larkins 2012).

Furthermore, the cupin comprises a ubiquitious protein superfamily characterized by the presence of a conserved barrel domain (Dunwell, 1998). This domain has two conserved motifs of $\beta$-strands separated by a less conserved region composed by another two $\beta$-strands with an intervening variable loop (Dunwell et al., 2000, 2001, 2002, 2003).

Cowpea 7S vicilins were found to contain two cupin_1 domains (bicupins), and $\beta$-vignins are the main representative of this protein class (Sales et al., 1992, 2001). Cowpea $\beta$-vignins associate in trimers that form a carbohydrate-binding multiprotein. Each monomer possesses an oligosaccharide interacting site that confers specific carbohydrate-binding property to the oligomeric structure (Dunwell et al., 2002, 2003). These binding sites are located at the vertices of the triangle-shaped oligomer and the interaction between $\beta$-vignins and oligosaccharides, mainly through hydrogen bond interactions (a typical feature of carbohydrate-protein interaction), was suggested by computational simulations (Rocha et al, 2018).

In this study the genome-wide database of cowpea genes was accessed in search of genomic sequences coding for cupins, given that its represents a well documented multigenic family of globulins. A total of seventy-seven genes belonging to the cupin superfamily were found, which were then classified into six families by phylogenetic reconstruction methods. V. unguiculata cupin genes were categorized into two groups: classical cupins (fifty-nine proteins) and bicupins (eighteen members). In addition, a search for cupin members on other related species the fabaceae family ( $V$. angularis, $V$. 
radiatam and Phaseolus vulgaris) was also performed. Based on these data, a detailed characterization and comparison of the cupin genes on the species analyzed was performed with the aim to better understand the connection and functions of cupin proteins from different, but related, plant sources.

\section{Methods}

\subsection{Dataset}

The $V$. unguiculata proteome IT97K-499-35 (genome assembly v1.0), available at the Phytozome database (http://phytozome.jgi.doe.gov/) (Goodstein et al., 2012), was accessed to search for proteins of the cupin superfamily. Furthermore, Rocha et al, 2018 were cloned six sequence denominate IT-81d-1053 (3R) and EPACE-10 3(S). 3 sequences resistance to $C$. maculatus and 3 susceptible to C. maculatus,

\subsection{Sequences analysis}

Analyses of the predicted the cupin superfamily proteins and identification of the cupin domain were performed using five different web servers. Pfam protein Database 2.0 (Finn et al, 2016), HMMER with Biosequence analysis using profile hidden Markov Models (Potter et al, 2018), SMART (Schultz et al, 2000) and Simple Modular Architecture Research Tool from the EMBL server and Conserved Domain tool from NCBI (CDD) (Marchler-Bauer., 2017). When applicable, only the result with the highest e-value was considered for analysis. BioEdit 7.2 software was used for edition (insertion and deletion) of amino acids sequences. The presence or absence of signal peptide was assessed with the SignalP 4,1 server (Petersen et al, 2011). The MEGA 7 (Tamakura et $a l, 2016)$ software was used for construction of phylogenetic tree using the NeighborJoining method (Saitou et al, 1987) with bootstrap values (1000 replicates).

\section{Protein structural model, docking and dynamic molecular}

Proteins structural modeling, docking, and dynamic molecular were performed essentially as described elsewhere (Rocha et al., 2018).

\section{Results and discussion}

\section{1- Cupin gene identification and analysis}

We identified 77 gene sequences encoding proteins containing one or two copies of the cupin superfamily domain in the genome of $V$. unguiculata (Table S1). These 
sequences were grouped into six cupin families (cupin-1 to cupin-5 and cupin-8), and no sequences related to cupin- 6 and cupin-7 families were found (Table S1).

The cupin-1 domain consists of a conserved barrel structure, and members of the cupin-1 family are represented by $11 \mathrm{~S}$ and $7 \mathrm{~S}$ seed storage globulins (termed legumins and vicilins, respectively) and germins (Dunwell et al, 1998). Legumins and vicilins are two-domain proteins (bicupins), whereas germins are single-domain molecules (monocupins) (Rocha et al, 2018) (Figure 1). $\beta$-vignins are the most abundant vicilins found in the genome of cowpea comprising 17 sequences (Table S 1 and 3), from which four are devoid of secretion signal peptides sequences: Vigun03g085800.1, Vigun03g085900.1, Vigun05g254700.1, vigun11g151800.1 (Table S2).

In a recent study based on computational simulations, Rocha et al (2018) demonstrated the presence of two cupin-1 domains in the primary structure of several $\beta$ vignin isoforms from two V. unguiculata genotypes (EPACE-10 and IT81D-1053) differing in the resistance to the bruchid beetle disease (Callosobrcuhus maculatus). In that study, the authors observed by computational simulations that $\beta$-vignin sequences presented a unique chitin-binding site (ChBS) in the $\mathrm{N}$-terminal and in $\mathrm{C}$-terminal ends (figure supp. 1). Those findings revealed the presence of three ChBS, which supports the hypothesis of the interaction of $V$. unguiculata $\beta$-vignins with the monosaccharide $\mathrm{N}$ Acetyl-D-Glucosamine (GlcNac) and possibly its oligomeric derivatives, as observed for other bicupins in the present study (figure S1).

Cupin families 2 and 4 were represented by only one sequence with one cupin domain each. Cupin families 3, 5 and 8 were represented, respectively, by 8,2 and 5 sequences with one cupin domain (Table S1 and 3).

We also identified sequences with 1 or 2 domains not belonging to the cupin-1 domain, such as auxin_BP, ARD, F-box-like, LacAB_rpiB. Some sequences contain one cupin domain and a second one not related to this superfamily. Sequences Vigun06g110700.1 (cupin-2 and LacAB_rpiB domain) and Vigun09g177900.1 (cupin-8 and F-box-like domains). Three sequences with domains Pirin and Pirin $\mathrm{C}$ were identified: Vigun03g399100.1, Vigun06g057200.1 and Vigun08g205900.1 (Table S1 and S3).

Other studies screening of the Capsicum annuum genome explored different proteins from the vicilin family, with attention to structural and functional features of Vic_capan sequences (vicilin of $C$. апnииm) revealing that those vicilins belong to the cupin superfamily. Vic capan are known by their multifunctional enzymatic roles, ranging 
from epimerase and transferase in prokaryotes to oxalate oxidase and iron-binding nuclear protein (pirin) in eukaryotes (Dunwell, et al 2014). In the genome of pea (Pisum sativum), there are at least 18 genes encoding $7 \mathrm{~S}$ vicilin divided into three small gene families, and 10 genes encoding legumins (Domoney and Casey 1985, Domoney et al. 1986). Furthermore, at least seven $11 \mathrm{~S}$ globulin genes have been described in soybean (Glycine max), and they are arranged in three groups based on amino acid identity (Beilinson et al. 2002). More than 100 cupin sequences have been identified in few plant species, like Oryza sativa, Vitis vinifera and Arabidopsis thaliana. This finding highlights the extent of which cupins have been duplicated and diverged throughout the evolution in genomes of plants to carry out several functions. In addition, Sreedhar et al. (2016) purified a protease from rice (Oryza sativa L.), which was further denominated cupincin. This protein was included as a new member of the cupin superfamily.

The $V$. unguiculata cupins were also inspected for the presence of a secretion signal peptide. A total of 52 from the 77 sequences contain a predicted signal peptide sequence, as per the SignalP web server. Several of those sequences share conserved regions (Table S1 and S2).

In addition, the presence of alternative transcripts derived from alternative splicing in the mRNA with primary structure in the cowpea genome was also investigated. Four genes were found (Vigun03g085800, Vigun03g085900, Vigun05g251000 and Vigun07g160600), each one presenting 2 alternative transcripts. Two of these transcripts (Vigun03g085800 and Vigun03g085900) encodes each one polypeptides with identical amino acid sequences, whereas the transcripts of Vigun05g251000 and Vigun07g160600 encodes different proteins. The gene Vigun05g251000.1 encodes a protein with 377 amino acid residues, whereas Vigun05g251000.2 encodes a shorter version (341 amino acid residues) of the same protein, lacking the first $36 \mathrm{~N}$-terminal residues in comparison to the larger isoform. The proteins encoded by Vigun07g160600 were identical, except in one isoform (Vigun07g160600.1; 226 amino acid residues), which was much longer than the other (Vigun07g160600.2; 222 amino acid residues), differing in 4 internal residues our insertion/deletion events.

In addition to $V$. unguiculata proteins, amino acid sequences belonging to other members of the cupin superfamily were also identified in closely related species: Vigna angularis, Vigna radiata and Phaseolus vulgaris (common bean) (Table S4 and S5

Eleven amino acid sequences of 7S vicilins, from which 10 are bicupins and one is a monocupin (ID: XP_017415489.1), were found in the genome of $V$. angularis. The 
same distribution pattern was found in $V$. radiata sequences, i.e. 10 sequences corresponding to bicupins and one monocupin, from a total of 11 sequences (Table S4 ). Regarding to $11 \mathrm{~S}$ legumins, only 4 amino acid sequences were identified from the databank of cowpea, all sequences being of the bicupin type (Table S4 ).

Three web servers were used to investigate the $P$. vulgaris genome: NCBI CDD for search for conserved domains, SMART and HMMER (Table S5). Twenty sequences were found with the CDD web server. From these sequences, 12 were monocupins and 3 were bicupins. The remaining 5 sequences were not related to the cupin superfamily; instead, they were identified as proteins associated to the glutelin provisional domain. The SMART web server returned 20 sequences, all of which contain the vicilin-1 domain and 9 of those with the cupin-1 domain. These findings were similar to the HMMER web server results, which led to the identification of 20 sequences (10 bicupins and 10 monocupins) (Table S5).

As discussed before, the high copy number of globulin genes is not exclusive to leguminous plants, being also reported in non-leguminous ones. For example, in Ficus pumila (Moraceae family), six 11S globulin isoforms have been reported (Chua et al. 2008). Similarly, also in hemp (Cannabis sativa L.), a member of Cannabaceae family that possess seven $11 \mathrm{~S}$ globulin (called edestin) genes, were identified and arranged into two groups (type1 and type2) based on differences on their primary structures (Docimo et al. 2014). The $11 \mathrm{~S}$ edestin is the main storage protein representing approximatively 80 $\%$ of the total seed protein, whereas approximatively $13 \%$ of the water-soluble protein are $2 \mathrm{~S}$ albumin.

\section{2-Molecular Phylogeny}

The phylogenetic classification of the cupin genes was performed initially only with sequences from $V$. unguiculata vicilins (Figure 2). Additionally, we analyzed sequences from 4 leguminous species from fabaceae family (V. unguiculata, V. angularis, $V$. radiata and $P$. vulgaris) (Figure 3 ). In the first phylogenetic tree, only 3 clades were identified between the $V$. unguiculata bicupins. The first clade includes 10 vicilin sequences obtained from cowpea database and 6 sequences of vicilins from cowpea with contrasting responses to the bruchid beetle (Callosobrcuhus maculatus) previously cloned by Rocha et al, 2018 (figure 2). In the second and third clades 4 and 5 vicilins are present, respectively, which correspond to the cupin_1 family. Others cupin families, 
202

203

204

205

206

207

208

209

210

211

212

213

214

215

216

217

218

219

220

221

222

223

224

225

226

227

228

229

230

231

232

233

234

such as cupin-2, 3, 4, 5, 7 and 8, are also present in this phylogenetic tree, as well as other sequences with non-cupin domain, such as Pirin-C, ARD and Axin_BP.

With respect to the analysis including the 4 selected plant species, the results showed that 3 clades were classified in accordance with position of the 19 amino acids sequences of bicupins of $V$. unguiculata (Figure 3). Again, clade 1 contains 9 bicupins obtained from the $V$. unguiculata database, as well as the 6 sequences cloned by Rocha et al (2018). Clade 2 contains 5 sequences from $V$. unguiculata bicupins. Clade 3 contains $4 V$. unguiculata bicupins and other bicupins from $V$. angulares, $V$. radiata and $P$. vulgaris (Figure 3).

All other amino acid sequences from $V$. angularis, $V$. radiata and Phaseolus vulgaris are bicupins that were not grouped with $V$. unguiculata bicupins. Moreover, the $34 \mathrm{~V}$. unguiculata monocupins identified (non-vicilins) form one larges clade. Three pirin domain-containing sequences (pirin and pirin $\mathrm{C}$ ), which belong to the cupin-2 family were also grouped in this analysis. Other research groups have studied human pirin sequences and have obtained their crystal structure, which is an iron-binding nuclear protein and transcription cofactor (Pang et al. (2004). In accordance with the first phylogenetic tree (Figure 1), in the second tree (Figura 3) also grouped cupin sequences belonging to the same family $(1,2,3,4,5,7$ and 8$)$, as well as sequences with no cupin domain, such as Pirin-C, ARD and Axin_BP.

\section{Conclusion}

In this study we identified six cupin families in the $V$. unguiculata genome, followed by the analysis of copy number of $7 \mathrm{~S}$ vicilins and/or $11 \mathrm{~S}$ legumin sequences from $V$.unguiculata and other leguminous plants, such as $V$. angularis, V. radiata, $P$. Vulgaris. This led to the identification of numerous cupin sequences in each species. Furthermore, the kind of and number of domains that are present in each sequences are described; and genes that are originated due the alternative splices are proposed. In summary, our results represent the first attempt at a thorough characterization of cupin sequences in important leguminous plants. This will support future studies to elucidate their biological role, which are still needed for a complete understanding of the cupin superfamily function, including $V$. unguiculata $\beta$-viginins with two cupin- 1 domains that are the major constituent of cowpea vicilins. 


\section{Acknowledgements}

This work was supported by grants from "Conselho Nacional de Desenvolvimento

Científico e Tecnológico" (CNPq) and "Coordenação de Aperfeiçoamento de Pessoal de

Nível Superior" (CAPES). AJR was recipient of Doctoral Fellowships from CAPES and

CNPq. AJR, ALSP and JEMJ wrote the manuscript. JHC done the phylogenetic tree.

\section{References}

Beilinson, V., Chen, Z., Shoemaker, R.C., Fischer, R.L.,Goldberg, R.B., Nielsen, N.C.:

Genomic organization of glycinin genes in soybean. - Theor. appl. Genet. 104: 11321140, 2002. families encoding 11S globulin and $2 \mathrm{~S}$ albumin isoforms of jelly fig (Ficus awkeotsang) achenes. - Biosci. Biotechnol. Biochem. 72: 506-513, 2008.

Domoney, C., Casey, R.: Measurement of gene number for seed storage proteins in Pisum. - Nucl. Acids Res. 13: 687-699, 1985.

Domoney, C., Ellis, T.H.N., Davies, D.R.: Organization and mapping of legumin genes in Pisum. - Mol. gen. Genet. 202: 280-285, 1986 characterization of edestin gene family in Cannabis sativa. - Plant. Physiol. Biochem. 84: $142-148$.

Dunwell, J.M., 1998. Cupins: a new superfamily of functionallydiverse proteins that include germins and plant seed storage proteins. Biotechnol. Genet. Engin. Rev. 15, 132.

Dunwell, J.M., Culham, A., Carter, C.E., Sosa-Aguirre, C.R., Goodenough, P.W., 2001. Evolution of functional diversity in the cupin superfamily. Trends Biochem. Sci. 26, 740-745.

263 Dunwell, J.M., Khuri, S., Gane, P.J., 2000. Microbial relatives of the seed storage proteins of higher plants: conservation of structure, and diversification of function during evolution of the cupin superfamily. Microbiol. Mol. Biol. Rev. 64, 153-179 Dunwell, J.M., 2002. Future prospects for transgenic crops. Phytochem. Rev. 1, 1-12. Dunwell, J.M., 2003. Structure, function and evolution of the legumin seed storage proteins. In: Steinbu“chel, A., Fahnestock, S.R. (Eds.) Biopolymers, Vol. 8. Polyamides and Complex Proteinaceous Materials II. Wiley-VCH, Weinheim, pp. 223-253. 
272 Finn, R.D., et al. (2016), The Pfam protein families database: towards a more sustainable future: Nucleic Acids Res. 2016 Jan 4; 44(Database issue): D279-D285. https://doi.org/101093/nar/gkv1344

275 Goodstein, M.D., et al., 2012. Phytozome: a comparative platform for green plant 276 genomics, Nucleic Acids Res. 201240 (D1): D1178-D1186.

277 Howe, R.W., Currie, J.E., 1964. Some laboratory observations on the rates of evelopment, mortality and oviposition of several species of Bruchidae breeding in stored pulses, Bull. Entomol. Res. 55 437, https://doi.org/10.1017/S0007485300049580 Marchler-Bauer, A., et al. 2017. "CDD/SPARCLE: functional classification of proteins via subfamily domain architectures, Nucleic Acids Res.45(D)200-3. Osborne, N.J., et al. Prevalence of challenge-proven IgE-mediated food allergy using population-based sampling and predetermined challenge criteria in infants. J Allergy Clin Immunol 2011; 127:668-676.e1- 2. https://doi.org/1016/j.jaci.2011.01.039

Pang, H., et al., 2014. Crystal Structure of Human Pirin . An iron-binding nuclear protein and transcription cofactor. 279: 1491-1498. https://doi.org/10.1074/jbc.M310022200. Petersen, T.N.,2011. SignalP 4.0: discriminating signal peptides from transmembrane regions. Nat Methods. 2011 Sep 29;8(10):785-6. doi: 10.1038/nmeth.1701 Kriz, A.L., (1999) 7S Globulins of Cereals. In: Shewry P.R., Casey R. (eds) Seed Proteins. Springer, Dordrecht. https://doi.org/10.1007/978-94-011-4431-5_20 Improving the production and utilization of cowpea as food and fodder, Field Crop Res. 84 169-177, https://doi.org/10.1016/S0378-4290(03)00148-5

297 Rocha, A.J., 2018. Cloning of cDNA sequences encoding cowpea (Vigna unguiculata) vicilins: Computational simulations suggest a binding mode of cowpea vicilins to chitin oligomers, International Journal of Biological Macromolecules, 117: 565-573 https://doi.org/10.1016/j.ijbiomac.2018.05.197. unguiculata) vicilins by pepsin, papain and bruchid (insect) midgut proteinases, Comp. 
Sales, M.P., Pimenta, P.P., Paes, N.S., Grossi-De-Sa, M.F., Xavier J., 2001.Vicilins (7S storage globulins) of cowpea (Vigna unguiculata) seeds bind to chitinous structures of the midgut of Callosobruchus maculatus (Coleoptera: Bruchidae) larvae, Braz. J. Med.

Biol. Res. 34: 27-34, https://doi.org/10.1590/S0100-879X2001000100003. phylogenetic trees. Molecular Biology and Evolution, 4:.406-425.

311 Shotwell, M.A., Larkins, B.A., 2012. Improvement of the protein quality of seeds by

312 genetic engineering. - In: Dennis, E.S., Lewellyn, D.J. (ed.): Molecular Approaches to

313 Crop Improvement. Pp. 33-61. Springer-Verlag, Wien - New York 2012.

314 Schultz, J., et al., 2000. SMART: A Web-based tool for the study of genetically mobile 315 domains. Nucleic Acids Res. 28: 231-234.

316 Sreedhar, R., Kaul, P., 2016. Cupincin: A Unique Protease Purified from Rice (Oryza sativa L.) Bran Is a New Member of the Cupin Superfamily.PLoS ONE 11(4): e0152819. doi:10.1371/journal. pone.0152819.

319 Tamakura, K. et al., 2016. MEGA7: molecular evolutionary genetics analysis using 320 maximum likelihood, evolutionary distance, and maximum parsimony methods.

321 Molecular Biology and Evolution 33 (7):1870-4.
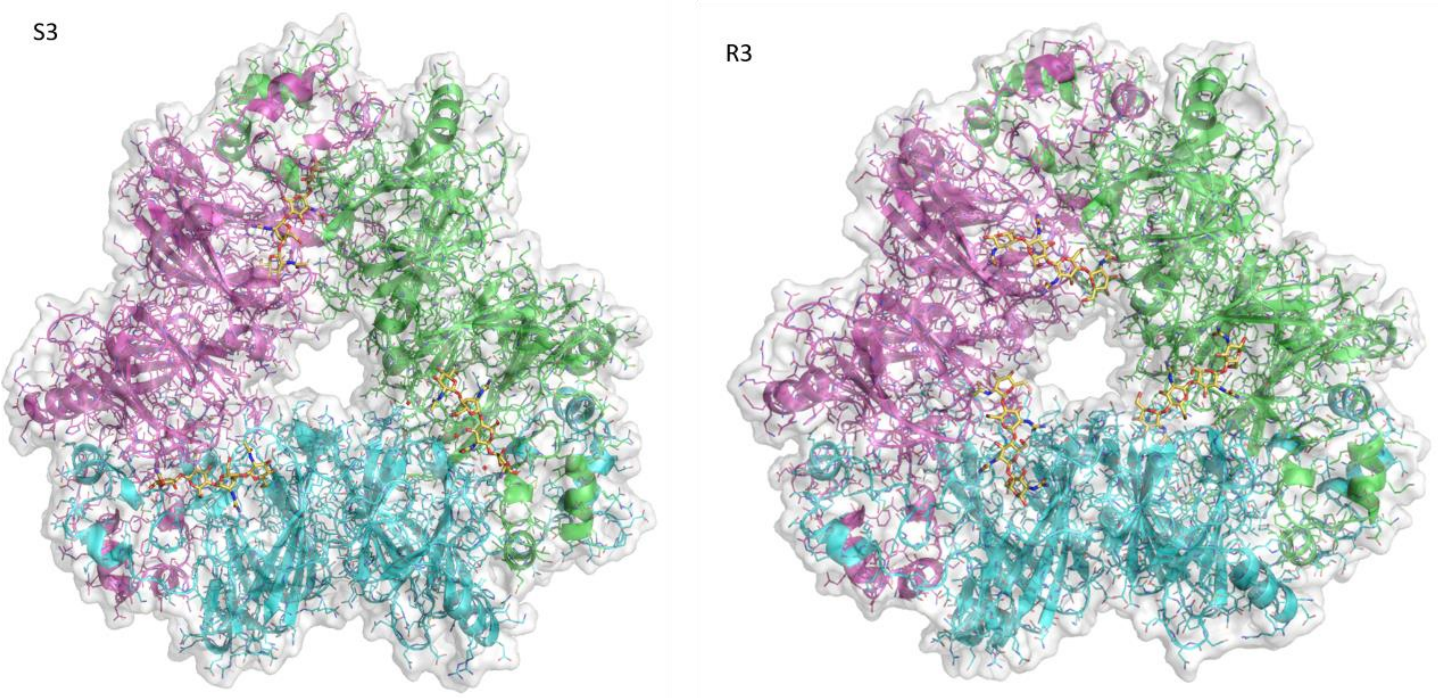
327 Figure 1- Ribbon diagrams of the $\beta$-vignin homotrimer models. Sequences Vicilin-S3-

328 MG973243.1 (A) and Vicilin-R3-MG973246 (B) are shown. Subunits are colored pink,

329 green and cyan. Chitooligosaccharide molecules [(GlcNAc) 4] docked in the chitin-

330 binding sites of each oligomer are also shown as stick models (carbon, nitrogen and

331 oxygen atoms are colored yellow, blue and red, respectively). For interpretation of the

332 references to color, the reader is referred to the web version of this article 
bioRxiv preprint doi: https://doi.org/10.1101/2020.06.07.138958; this version posted June 8, 2020. The copyright holder for this preprint (which was not certified by peer review) is the author/funder. All rights reserved. No reuse allowed without permission.

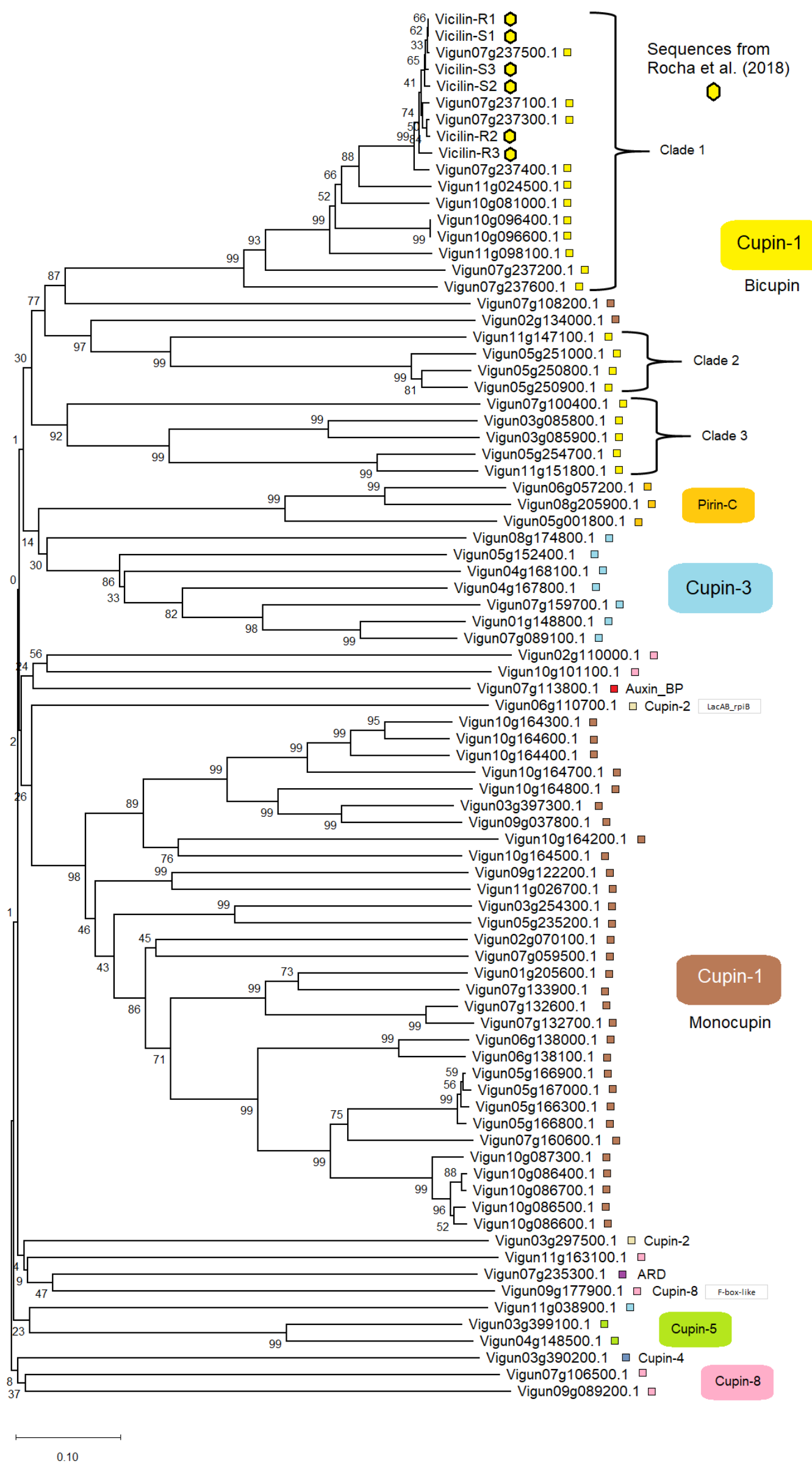


Figure2- Molecular phylogeny of $V$. unguiculata cupins. The different cupin families are highlighted in several colors and include monocupin and bicipuns (vicilins).

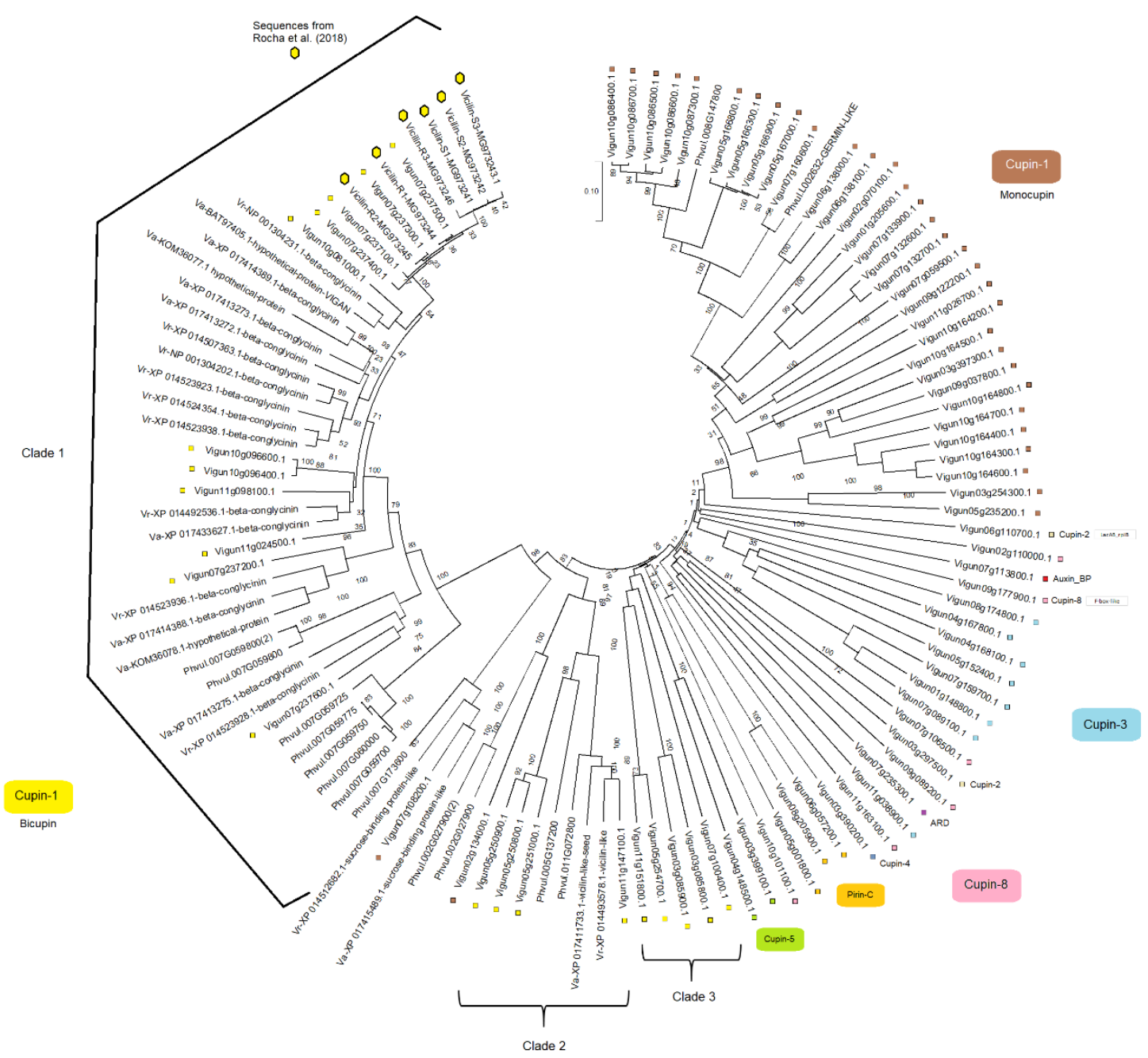

Figure 3- Molecular phylogeny of $V$. unguiculata, $V$. angulares, $V$. radiata and $P$. family, as well clades 2 and 3 that were also identified. The largest group in terms of $V$. unguiculata sequences is the one formed with cupin-1 monocupins. 


\section{SUPPLEMENTARY MATERIAL}

\section{Genome-wide analysis of the cupin superfamily in cowpea (Vigna unguiculata)}

\section{Antônio J. Rocha ${ }^{1 *}$, Mario Ramos de Oliveira Barsottini ${ }^{2}$ Ana Luiza Sobral Paiva ${ }^{1}$, José Hélio Costa ${ }^{1}$ Thalles Barbosa Grangeiro $^{1}$}

${ }^{1}$ Departamento de Bioquímica e Biologia Molecular, Centro de Ciências, Campus do Pici, Universidade Federal do Ceará, Fortaleza, Ceará,

$$
\text { 60.440-900, Brazil }
$$

${ }^{2}$ Laboratory of Genome e BioEnergy-LGE. Institute of Biology, Campinas State University, Campinas, São Paulo, Brazil

${ }^{3}$ Laboratório de Genética Molecular, Departamento de Biologia, Centro de Ciências, Campus do Pici, Universidade Federal do Ceará, Fortaleza,

Ceará, 60.440-900, Brazil

*To whom all correspondence should be addressed

E-mail: antonionubis@gmail.com

Table S1. Cupin superfamily proteins from cowpea (Vigna unguiculata)

\begin{tabular}{|c|c|c|c|c|c|c|c|c|c|c|}
\hline \multirow[b]{2}{*}{ Gene } & \multirow[b]{2}{*}{ Encoded protein } & \multirow{2}{*}{$\begin{array}{l}\text { Size } \\
\text { (residues) }\end{array}$} & \multirow[b]{2}{*}{$\begin{array}{l}\text { Molecular } \\
\text { mass (Da) }\end{array}$} & \multirow{2}{*}{$\begin{array}{l}\text { Theoretical } \\
\text { pI }\end{array}$} & \multirow{2}{*}{$\begin{array}{l}\text { Signal } \\
\text { peptide } \\
\text { (start-end) }\end{array}$} & \multicolumn{5}{|c|}{ Cupin domain(s) } \\
\hline & & & & & & PFAM family & Accession & Start-End & $\begin{array}{c}\text { Individual } \\
\text { E-value }\end{array}$ & $\begin{array}{c}\text { Confidential } \\
\text { E-value }\end{array}$ \\
\hline Vigun01g148800.1 & $\begin{array}{l}\text { Protein of unknown } \\
\text { function (DUF861) }\end{array}$ & 110 & 12041.65 & 8.27 & No & Cupin_3 & PF05899.11 & $22-96$ & $4.0 e-26$ & $2.4 \mathrm{e}-30$ \\
\hline Vigun01g205600.1 & Cupin & 217 & 22824.33 & 8.52 & $1-21$ & Cupin_1 & PF00190.21 & $60-207$ & $7.1 \mathrm{e}-48$ & $8.5 e-52$ \\
\hline Vigun02g070100.1 & Cupin & 216 & 23407.83 & 8.49 & $1-21$ & Cupin_1 & PF00190.21 & $59-208$ & $1.4 \mathrm{e}-39$ & $1.7 e-43$ \\
\hline Vigun02g110000.1 & Cupin-like domain & 415 & 47334.28 & 5.64 & $1-19$ & Cupin_8 & PF13621.5 & $100-377$ & $4.4 \mathrm{e}-76$ & $2.7 e-80$ \\
\hline Vigun02g134000.1 & Cupin & 631 & 73379.36 & 5.25 & $1-26$ & Cupin_1 & PF00190.21 & 238-393 & $1.8 \mathrm{e}-32$ & $1.1 \mathrm{e}-36$ \\
\hline
\end{tabular}




\begin{tabular}{|c|c|c|c|c|c|c|c|c|c|c|}
\hline \multirow{2}{*}{ Vigun03g085800.1 } & \multirow{2}{*}{$\begin{array}{l}\text { Cupin } \\
\text { Cupin }\end{array}$} & \multirow{2}{*}{357} & \multirow{2}{*}{38679.55} & \multirow{2}{*}{5.23} & \multirow{2}{*}{ No } & \multirow{2}{*}{$\begin{array}{l}\text { Cupin_1 } \\
\text { Cupin_1 }\end{array}$} & PF00190.21 & $5-159$ & $5.5 e-21$ & $6.6 e-25$ \\
\hline & & & & & & & PF00190.21 & $192-340$ & $6.5 e-19$ & $7.8 e-23$ \\
\hline \multirow{2}{*}{ Vigun03g085900.1 } & \multirow{2}{*}{$\begin{array}{l}\text { Cupin } \\
\text { Cupin }\end{array}$} & \multirow{2}{*}{358} & \multirow{2}{*}{38785.50} & \multirow{2}{*}{5.00} & \multirow{2}{*}{ No } & \multirow{2}{*}{$\begin{array}{l}\text { Cupin_1 } \\
\text { Cupin_1 }\end{array}$} & PF00190.21 & $5-159$ & $7.7 e-21$ & $9.2 \mathrm{e}-25$ \\
\hline & & & & & & & PF00190.21 & $192-341$ & $1.0 \mathrm{e}-14$ & $1.2 \mathrm{e}-18$ \\
\hline Vigun03g254300.1 & Cupin & 155 & 16831.42 & 9.43 & No & Cupin_1 & PF00190.21 & $5-140$ & $2.4 \mathrm{e}-28$ & $2.8 e-32$ \\
\hline Vigun03g297500.1 & Cupin domain & 297 & 33408.29 & 5.98 & $1-19$ & Cupin_2 & PF07883.10 & $220-289$ & $2.0 e-06$ & $2.4 \mathrm{e}-10$ \\
\hline Vigun03g390200.1 & $\begin{array}{l}\text { Cupin superfamily } \\
\text { protein }\end{array}$ & 776 & 87862.48 & 8.40 & $1-17$ & Cupin_4 & PF08007.11 & $439-575$ & $6.8 e-25$ & $4.1 e-29$ \\
\hline Vigun03g397300.1 & Cupin & 208 & 21325.81 & 7.79 & No & Cupin_1 & PF00190.21 & $52-197$ & $2.3 e-35$ & $2.8 e-39$ \\
\hline Vigun03g399100.1 & $\begin{array}{l}\text { Cupin superfamily } \\
\text { (DUF985) }\end{array}$ & 182 & 21222.94 & 5.42 & No & Cupin_5 & PF06172.10 & $6-166$ & $4.7 e-42$ & $2.8 e-46$ \\
\hline Vigun04g148500.1 & $\begin{array}{l}\text { Protein of unknown } \\
\text { function (DUF861) }\end{array}$ & 98 & 20202.80 & 5.09 & No & Cupin_3 & PF06172.10 & $6-159$ & $5.2 \mathrm{e}-41$ & $3.1 e-45$ \\
\hline Vigun04g167800.1 & $\begin{array}{l}\text { Cupin superfamily } \\
\text { (DUF985) }\end{array}$ & 189 & 11067.81 & 7.62 & No & Cupin_5 & PF05899.11 & $21-95$ & $1.9 \mathrm{e}-27$ & $1.1 \mathrm{e}-31$ \\
\hline Vigun04g168100.1 & $\begin{array}{l}\text { Protein of unknown } \\
\text { function (DUF861) }\end{array}$ & 129 & 14501.24 & 9.10 & $1-31$ & Cupin_3 & PF05899.11 & $51-125$ & $6.6 e-24$ & $7.9 e-28$ \\
\hline \multirow{2}{*}{ Vigun05g001800.1 } & \multirow{2}{*}{$\begin{array}{l}\text { Pirin } \\
\text { Pirin C-terminal } \\
\text { cupin domain } \\
\end{array}$} & \multirow[b]{2}{*}{322} & \multirow[b]{2}{*}{35815.78} & \multirow[b]{2}{*}{6.31} & \multirow[b]{2}{*}{ No } & \multirow{2}{*}{$\begin{array}{l}\text { Pirin } \\
\text { Pirin_C }\end{array}$} & PF02678.15 & $59-354$ & $4.3 e-33$ & $5.1 \mathrm{e}-37$ \\
\hline & & & & & & & PF05726.12 & $207-312$ & $1.3 e-31$ & $1.6 e-35$ \\
\hline Vigun05g152400.1 & $\begin{array}{l}\text { Protein of unknown } \\
\text { function (DUF861) }\end{array}$ & 58 & 6736.85 & 9.25 & No & Cupin_3 & PF05899.11 & $21-58$ & $4.3 e-09$ & $2.6 e-13$ \\
\hline Vigun05g166300.1 & Cupin & 222 & 24032.52 & 8.51 & $1-20$ & Cupin_1 & PF00190.21 & $60-210$ & $2.4 \mathrm{e}-47$ & $2.9 e-51$ \\
\hline Vigun05g166800.1 & Cupin & 222 & 23998.50 & 8.51 & $1-20$ & Cupin_1 & PF00190.21 & $60-210$ & $3.3 e-47$ & $3.9 e-51$ \\
\hline Vigun05g166900.1 & Cupin & 222 & 23983.53 & 8.51 & $1-20$ & Cupin_1 & PF00190.21 & $60-210$ & $6.1 \mathrm{e}-47$ & $7.2 e-51$ \\
\hline Vigun05g167000.1 & Cupin & 222 & 24051.56 & 8.51 & $1-26$ & Cupin_1 & PF00190.21 & $60-210$ & $6.7 e-46$ & $8.0 e-50$ \\
\hline Vigun05g235200.1 & Cupin & 204 & 21833.80 & 6.26 & $1-20$ & Cupin_1 & PF00190.21 & $53-196$ & $5.0 e-31$ & $5.9 e-35$ \\
\hline \multirow{2}{*}{ Vigun05g250800.1 } & \multirow{2}{*}{$\begin{array}{l}\text { Cupin } \\
\text { Cupin }\end{array}$} & \multirow{2}{*}{456} & \multirow{2}{*}{50920.54} & \multirow{2}{*}{4.99} & \multirow{2}{*}{$1-19$} & \multirow{2}{*}{$\begin{array}{l}\text { Cupin_1 } \\
\text { Cupin_1 }\end{array}$} & PF00190.21 & $43-192$ & $1.8 \mathrm{e}-10$ & $2.2 \mathrm{e}-14$ \\
\hline & & & & & & & PF00190.21 & $292-441$ & $3.8 \mathrm{e}-27$ & $4.6 e-31$ \\
\hline \multirow{2}{*}{ Vigun05g250900.1 } & \multirow{2}{*}{$\begin{array}{l}\text { Cupin } \\
\text { Cupin }\end{array}$} & \multirow{2}{*}{456} & 5117889 & 5.14 & $1-10$ & Cupin_1 & PF00190.21 & $43-194$ & $6.0 \mathrm{e}-13$ & $7.2 \mathrm{e}-17$ \\
\hline & & & $511 / 8.89$ & 5.14 & $1-19$ & Cupin_1 & PF00190.21 & 292 & $3.8 \mathrm{e}-27$ & $4.6 e-31$ \\
\hline Vigun050751000 1 & Cupin & 377 & 4262097 & 520 & $1-19$ & Cupin_1 & PF00190.21 & $2-113$ & $1.1 \mathrm{e}-08$ & $6.3 e-13$ \\
\hline vigunusgzsiv00.1 & Cupin & $3 / 1$ & 42020.91 & 5.20 & $1-19$ & Cupin_1 & PF00190.21 & $213-262$ & $1.8 \mathrm{e}-25$ & $1.1 \mathrm{e}-29$ \\
\hline
\end{tabular}




\begin{tabular}{|c|c|c|c|c|c|c|c|c|c|c|}
\hline \multirow{2}{*}{ Vigun05g254700.1 } & \multirow{2}{*}{$\begin{array}{l}\text { Cupin } \\
\text { Cupin }\end{array}$} & \multirow{2}{*}{356} & \multirow{2}{*}{38144.76} & \multirow{2}{*}{5.66} & \multirow{2}{*}{ No } & \multirow{2}{*}{$\begin{array}{l}\text { Cupin_1 } \\
\text { Cupin_1 }\end{array}$} & \multirow{2}{*}{$\begin{array}{l}\text { PF00190.21 } \\
\text { PF00190.21 }\end{array}$} & \multirow{2}{*}{$\begin{array}{r}6-157 \\
190-339\end{array}$} & \multirow{2}{*}{\begin{tabular}{|l|}
$3.6 e-27$ \\
$1.7 e-22$ \\
\end{tabular}} & \multirow{2}{*}{$\begin{array}{l}2.2 \mathrm{e}-31 \\
1.0 \mathrm{e}-26 \\
\end{array}$} \\
\hline & & & & & & & & & & \\
\hline Vigun06g057200.1 & $\begin{array}{l}\text { Pirin C- } \\
\text { Cupin domain }\end{array}$ & 298 & 38144.76 & 5.66 & No & $\begin{array}{l}\text { Pirin } \\
\text { Pirin_C }\end{array}$ & $\begin{array}{l}\text { PF02678.15 } \\
\text { PF05726.12 }\end{array}$ & $\begin{array}{c}30-126 \\
179-284\end{array}$ & $\begin{array}{l}1.8 e-32 \\
3.7 e-31\end{array}$ & $\begin{array}{l}2.1 e-36 \\
4.5 e-35\end{array}$ \\
\hline Vigun06g110700.1 & $\begin{array}{l}\text { Ribose/Galactose } \\
\text { Isomerase } \\
\text { Cupin domain }\end{array}$ & 302 & 32834.09 & 5.09 & No & $\begin{array}{l}\text { LacAB_rpiB } \\
\text { Cupin_2 }\end{array}$ & $\begin{array}{l}\text { PF02502.17 } \\
\text { PF07883.10 }\end{array}$ & $\begin{array}{c}11-139 \\
202-269\end{array}$ & $\begin{array}{l}3.5 e-31 \\
1.1 e-07\end{array}$ & $\begin{array}{l}2.1 e-36 \\
4.5 e-35\end{array}$ \\
\hline Vigun06g138000.1 & Cupin & 215 & 22973.39 & 6.03 & $1-19$ & Cupin_1 & PF00190.21 & $59-209$ & $1.2 \mathrm{e}-45$ & $1.4 \mathrm{e}-49$ \\
\hline Vigun06g138100.1 & Cupin & 214 & 23021.51 & 6.03 & $1-20$ & Cupin_1 & PF00190.21 & $59-209$ & $6.9 e-47$ & $8.2 e-51$ \\
\hline Vigun07g059500.1 & Cupin & 225 & 24154.63 & 6.59 & $1-23$ & Cupin_1 & PF00190.21 & $67-213$ & $2.7 e-42$ & $3.2 \mathrm{e}-46$ \\
\hline Vigun07g089100.1 & $\begin{array}{l}\text { Protein of unknown } \\
\text { function (DUF861) }\end{array}$ & 104 & 11564.11 & 6.71 & $1-23$ & Cupin_3 & PF05899.11 & $22-96$ & $3.2 e-27$ & $1.9 e-31$ \\
\hline \multirow{2}{*}{ Vigun07g100400.1 } & Cupin & \multirow{2}{*}{611} & \multirow{2}{*}{69379.61} & \multirow{2}{*}{5.30} & \multirow{2}{*}{$1-20$} & \multirow{2}{*}{$\begin{array}{l}\text { Cupin_1 } \\
\text { Cupin_1 }\end{array}$} & PF00190.21 & $39-192$ & $2.0 \mathrm{e}-25$ & $1.2 \mathrm{e}-29$ \\
\hline & Cupin & & & & & & PF00190.21 & $443-589$ & $1.0 \mathrm{e}-30$ & $6.3 e-35$ \\
\hline Vigun07g106500.1 & Cupin-like domain & 537 & 61142.18 & 5.27 & No & Cupin_8 & PF13621.5 & $18-295$ & $1.4 \mathrm{e}-47$ & $1.7 e-51$ \\
\hline Vigun07g108200.1 & Cupin & 510 & 57790.61 & 6.00 & $1-26$ & Cupin_1 & PF00190.21 & $320-480$ & $7.7 e-26$ & $4.6 e-30$ \\
\hline Vigun07g113800.1 & $\begin{array}{l}\text { Auxin binding } \\
\text { protein }\end{array}$ & 192 & 21867.96 & 5.76 & $1-23$ & Auxin_BP & PF02041.15 & 24-192 & $1.3 e-97$ & $1.6 e-101$ \\
\hline Vigun07g132600.1 & Cupin & 220 & 23203.58 & 6.82 & $1-22$ & Cupin_1 & PF00190.21 & $61-209$ & $9.2 e-49$ & $1.1 \mathrm{e}-52$ \\
\hline Vigun07g132700.1 & Cupin & 220 & 23246.70 & 7.64 & $1-22$ & Cupin_1 & PF00190.21 & $61-209$ & $2.1 \mathrm{e}-48$ & $2.5 e-52$ \\
\hline Vigun07g133900.1 & Cupin & 218 & 23121.54 & 8.53 & $1-22$ & Cupin_1 & PF00190.21 & $60-208$ & $5.6 e-48$ & $6.7 e-52$ \\
\hline Vigun07g159700.1 & $\begin{array}{l}\text { Protein of unknown } \\
\text { function (DUF861) }\end{array}$ & 115 & 12642.45 & 8.91 & $1-20$ & Cupin_3 & PF05899.11 & $26-100$ & $7.2 e-29$ & $4.3 e-33$ \\
\hline Vigun07g160600.1 & Cupin & 226 & 24302.96 & 6.81 & $1-20$ & Cupin_1 & PF00190.21 & $65-215$ & $9.0 e-47$ & $1.1 \mathrm{e}-50$ \\
\hline Vigun07g235300.1 & ARD/ARD' family & 187 & 22269.20 & 5.05 & No & ARD & PF03079.13 & $4-158$ & $2.3 e-61$ & $4.0 e-65$ \\
\hline \multirow{2}{*}{ Vigun07g237100.1 } & Cupin & \multirow{2}{*}{444} & \multirow{2}{*}{51037.19} & \multirow{2}{*}{5.33} & \multirow{2}{*}{$1-23$} & Cupin_1 & PF00190.21 & $56-206$ & $5.4 \mathrm{e}-08$ & $3.2 \mathrm{e}-12$ \\
\hline & Cupin & & & & & Cupin_1 & PF00190.21 & $258-417$ & $8.6 e-27$ & 5.1e-31 \\
\hline \multirow{2}{*}{ Vigun07g237200.1 } & Cupin & \multirow{2}{*}{536} & \multirow{2}{*}{61804.46} & \multirow{2}{*}{5.92} & \multirow{2}{*}{$1-23$} & Cupin_1 & PF00190.21 & $140-288$ & 0.00011 & $6.5 e-09$ \\
\hline & Cupin & & & & & Cupin_1 & PF00190.21 & $341-496$ & $8.9 e-24$ & $5.3 e-28$ \\
\hline \multirow{2}{*}{ Vigun07g237300.1 } & Cupin & \multirow{2}{*}{457} & \multirow{2}{*}{52584.93} & \multirow{2}{*}{5.40} & $1-25$ & Cupin_1 & PF00190.21 & $56-206$ & $5.7 e-08$ & $3.4 \mathrm{e}-12$ \\
\hline & Cupin & & & & $1-\angle 3$ & Cupin_1 & PF00190.21 & $258-417$ & $9.1 \mathrm{e}-27$ & $5.5 e-31$ \\
\hline
\end{tabular}




\begin{tabular}{|c|c|c|c|c|c|c|c|c|c|c|}
\hline \multirow{2}{*}{ Vigun07g237400.1 } & \multirow{2}{*}{$\begin{array}{l}\text { Cupin } \\
\text { Cupin }\end{array}$} & \multirow{2}{*}{442} & \multirow{2}{*}{50774.80} & \multirow{2}{*}{5.33} & \multirow{2}{*}{$1-23$} & \multirow{2}{*}{$\begin{array}{l}\text { Cupin_1 } \\
\text { Cupin_1 }\end{array}$} & PF00190.21 & 54-204 & $6.0 \mathrm{e}-08$ & $3.6 e-12$ \\
\hline & & & & & & & PF00190.21 & $256-415$ & $8.5 e-27$ & $5.1 \mathrm{e}-31$ \\
\hline \multirow{2}{*}{ Vigun07g237500.1 } & \multirow{2}{*}{$\begin{array}{l}\text { Cupin } \\
\text { Cupin }\end{array}$} & \multirow{2}{*}{455} & \multirow{2}{*}{52225.44} & \multirow{2}{*}{5.33} & \multirow{2}{*}{$1-23$} & \multirow{2}{*}{$\begin{array}{l}\text { Cupin_1 } \\
\text { Cupin_1 }\end{array}$} & PF00190.21 & $54-204$ & $5.1 \mathrm{e}-08$ & $3.1 \mathrm{e}-12$ \\
\hline & & & & & & & PF00190.21 & $256-415$ & $9.0 \mathrm{e}-27$ & $5.4 \mathrm{e}-31$ \\
\hline Vigun07g237600.1 & Cupin & 589 & 69269.70 & 6.50 & $1-24$ & Cupin_1 & PF00190.21 & $395-551$ & $1.5 e-25$ & $8.7 e-30$ \\
\hline Vigun08g174800.1 & $\begin{array}{l}\text { Protein of unknown } \\
\text { function (DUF861) }\end{array}$ & 143 & 16551.91 & 9.18 & No & Cupin_3 & PF05899.11 & $61-135$ & $6.9 e-20$ & $4.1 \mathrm{e}-24$ \\
\hline \multirow[b]{2}{*}{ Vigun08g205900.1 } & \multirow{2}{*}{$\begin{array}{l}\text { Pirin } \\
\text { Pirin C-terminal } \\
\text { cupin domain }\end{array}$} & \multirow[b]{2}{*}{301} & \multirow[b]{2}{*}{33819.51} & \multirow[b]{2}{*}{5.95} & \multirow[b]{2}{*}{ No } & \multirow{2}{*}{$\begin{array}{c}\text { Pirin } \\
\text { Pirin_C }\end{array}$} & PF02678.15 & $30-126$ & $3.9 e-32$ & $4.7 e-36$ \\
\hline & & & & & & & PF05726.12 & $179-284$ & $6.7 e-34$ & $8.0 e-38$ \\
\hline Vigun09g037800.1 & Cupin & 209 & 21717.89 & 5.35 & $1-18$ & Cupin_1 & PF00190.21 & 53-199 & $5.9 e-36$ & $7.0 \mathrm{e}-40$ \\
\hline Vigun09g089200.1 & Cupin-like domain & 483 & 56165.26 & 5.14 & No & Cupin_8 & PF13621.5 & $19-291$ & $2.3 e-22$ & $2.8 \mathrm{e}-26$ \\
\hline Vigun09g122200.1 & Cupin & 231 & 25405.93 & 6.74 & $1-25$ & Cupin_1 & PF00190.21 & $66-202$ & $1.0 \mathrm{e}-26$ & $1.2 \mathrm{e}-30$ \\
\hline \multirow{2}{*}{ Vigun09g177900.1 } & \multirow{2}{*}{$\begin{array}{l}\text { F-box-like } \\
\text { Cupin-like domain }\end{array}$} & \multirow{2}{*}{962} & \multirow{2}{*}{110138.09} & \multirow{2}{*}{5.42} & \multirow{2}{*}{ No } & \multirow{2}{*}{$\begin{array}{l}\text { F-box-like } \\
\text { Cupin_8 }\end{array}$} & PF12937.6 & $15-61$ & $5.5 e-08$ & $1.3 e-11$ \\
\hline & & & & & & & PF13621.5 & $135-367$ & $1.8 \mathrm{e}-20$ & $4.4 \mathrm{e}-24$ \\
\hline \multirow{2}{*}{ Vigun10g081000.1 } & Cupin & 457 & 5275717 & 531 & $1-23$ & Cupin_1 & PF00190.21 & $51-204$ & $1.8 \mathrm{e}-06$ & $1.1 \mathrm{e}-10$ \\
\hline & Cupin & $45 /$ & & 5.31 & $1-23$ & Cupin_1 & PF00190.21 & $254-417$ & $6.9 \mathrm{e}-26$ & $4.2 \mathrm{e}-30$ \\
\hline Vigun10g086400.1 & Cupin & 220 & 23724.33 & 6.06 & $1-20$ & Cupin_1 & PF00190.21 & $60-209$ & $4.7 e-47$ & $5.6 e-51$ \\
\hline Vigun10g086500.1 & Cupin & 220 & 23700.31 & 6.06 & $1-20$ & Cupin_1 & PF00190.21 & $60-209$ & $3.7 e-47$ & $4.4 e-51$ \\
\hline Vigun10g086600.1 & Cupin & 220 & 23679.42 & 6.90 & $1-20$ & Cupin_1 & PF00190.21 & $62-209$ & $2.3 e-46$ & $2.8 \mathrm{e}-50$ \\
\hline Vigun10g086700.1 & Cupin & 220 & 23682.29 & 6.06 & $1-20$ & Cupin_1 & PF00190.21 & $60-209$ & $6.6 e-47$ & $7.9 e-51$ \\
\hline Vigun10g087300.1 & Cupin & 220 & 23655.18 & 5.77 & $1-20$ & Cupin_1 & PF00190.21 & $60-209$ & $6.2 \mathrm{e}-47$ & $7.5 e-51$ \\
\hline Vigun10g096400.1 & Cupin & 456 & 52579.95 & 5.38 & $1-23$ & Cupin_1 & PF00190.21 & $54-204$ & 4.7e-09 & $2.8 \mathrm{e}-13$ \\
\hline VIgun IUg096400.1 & Cupin & 456 & 52519.95 & 5.58 & $1-23$ & Cupin_1 & PF00190.21 & $256-423$ & $3.2 \mathrm{e}-25$ & $1.9 \mathrm{e}-29$ \\
\hline & Cupin & & 5257995 & 538 & & Cupin_1 & PF00190.21 & $54-204$ & 4.7e-09 & $2.8 \mathrm{e}-13$ \\
\hline VIgun10g096600.1 & Cupin & 456 & $525 / 9.95$ & 5.38 & $1-23$ & Cupin_1 & PF00190.21 & $256-423$ & $3.2 \mathrm{e}-25$ & $1.9 \mathrm{e}-29$ \\
\hline Vigun10g101100.1 & Cupin-like domain & 516 & 58866.96 & 6.51 & No & Cupin_8 & PF13621.5 & $214-439$ & $5.1 \mathrm{e}-22$ & $6.1 \mathrm{e}-26$ \\
\hline Vigun10g164200.1 & Cupin & 209 & 22761.19 & 9.01 & $1-20$ & Cupin_1 & PF00190.21 & 57-199 & $4.5 e-15$ & $5.3 e-19$ \\
\hline Vigun10g164300.1 & Cupin & 209 & 21899.21 & 6.81 & $1-20$ & Cupin_1 & PF00190.21 & 54-199 & $1.9 \mathrm{e}-34$ & $2.3 e-38$ \\
\hline Vigun10g164400.1 & Cupin & 206 & 21694.07 & 6.50 & $1-17$ & Cupin_1 & PF00190.21 & $51-196$ & $6.0 \mathrm{e}-34$ & $7.2 \mathrm{e}-38$ \\
\hline Vigun10g164500.1 & Cupin & 212 & 22565.74 & 5.12 & $1-23$ & Cupin_1 & PF00190.21 & $60-202$ & $3.0 \mathrm{e}-22$ & $3.6 e-26$ \\
\hline Vigun10g164600.1 & Cupin & 213 & 22353.56 & 6.39 & $1-20$ & Cupin_1 & PF00190.21 & $54-199$ & $6.8 e-35$ & $8.2 e-39$ \\
\hline
\end{tabular}




\begin{tabular}{|c|c|c|c|c|c|c|c|c|c|c|}
\hline Vigun10g164700.1 & Cupin & 206 & 21578.92 & 7.81 & $1-17$ & Cupin_1 & PF00190.21 & $52-196$ & $2.6 \mathrm{e}-31$ & $3.2 \mathrm{e}-35$ \\
\hline Vigun10g164800.1 & Cupin & 209 & 21875.32 & 6.64 & $1-18$ & Cupin_1 & PF00190.21 & 53-199 & $3.3 e-29$ & $2.0 \mathrm{e}-33$ \\
\hline \multirow{2}{*}{ Vigun11g024500.1 } & \multirow{2}{*}{$\begin{array}{l}\text { Cupin } \\
\text { Cupin }\end{array}$} & \multirow{2}{*}{449} & \multirow{2}{*}{51413.94} & \multirow{2}{*}{5.96} & \multirow{2}{*}{$1-23$} & \multirow{2}{*}{$\begin{array}{l}\text { Cupin_1 } \\
\text { Cupin_1 }\end{array}$} & PF00190.21 & $44-201$ & $1.8 \mathrm{e}-06$ & $1.1 \mathrm{e}-10$ \\
\hline & & & & & & & PF00190.21 & $249-410$ & $7.5 e-26$ & $4.5 e-30$ \\
\hline Vigun11g026700.1 & Cupin & 228 & 24995.64 & 6.06 & $1-26$ & Cupin_1 & PF00190.21 & $67-211$ & $1.2 \mathrm{e}-27$ & $1.4 \mathrm{e}-31$ \\
\hline Vigun11g038900.1 & $\begin{array}{l}\text { Protein of unknown } \\
\text { function (DUF861) }\end{array}$ & 206 & 23763.62 & 8.85 & $1-26$ & Cupin_3 & PF05899.11 & $169-206$ & $2.8 \mathrm{e}-07$ & $1.7 e-11$ \\
\hline \multirow{2}{*}{ Vigun11g098100.1 } & Cupin & \multirow{2}{*}{427} & \multirow{2}{*}{49123.27} & \multirow{2}{*}{5.14} & \multirow{2}{*}{$1-23$} & \multirow{2}{*}{$\begin{array}{l}\text { Cupin_1 } \\
\text { Cupin_1 }\end{array}$} & PF00190.21 & $51-202$ & $1.5 \mathrm{e}-07$ & $1.8 \mathrm{e}-11$ \\
\hline & Cupin & & & & & & PF00190.21 & $252-409$ & $6.4 \mathrm{e}-25$ & $7.6 e-29$ \\
\hline \multirow{2}{*}{ Vigun11g147100.1 } & Cupin & \multirow{2}{*}{444} & \multirow{2}{*}{49794.57} & \multirow{2}{*}{5.21} & \multirow{2}{*}{$1-21$} & \multirow{2}{*}{$\begin{array}{l}\text { Cupin_1 } \\
\text { Cupin_1 }\end{array}$} & PF00190.21 & $31-146$ & $1.4 \mathrm{e}-10$ & $1.6 \mathrm{e}-14$ \\
\hline & Cupin & & & & & & PF00190.21 & 275 & $1.5 e-29$ & $1.8 \mathrm{e}-33$ \\
\hline \multirow{2}{*}{ Vigun11g151800.1 } & Cupin & \multirow{2}{*}{356} & \multirow{2}{*}{38458.15} & \multirow{2}{*}{6.38} & \multirow{2}{*}{ No } & \multirow{2}{*}{$\begin{array}{l}\text { Cupin_1 } \\
\text { Cupin_1 }\end{array}$} & PF00190.21 & 5-157 & $3.1 \mathrm{e}-29$ & $1.9 \mathrm{e}-33$ \\
\hline & Cupin & & & & & & PF00190.21 & $190-339$ & $2.4 \mathrm{e}-22$ & $1.5 \mathrm{e}-26$ \\
\hline Vigun11g163100.1 & Cupin-like domain & 413 & 46056.06 & 5.58 & No & Cupin_8 & PF13621.5 & $180-410$ & $1.6 \mathrm{e}-46$ & $2.8 \mathrm{e}-50$ \\
\hline
\end{tabular}




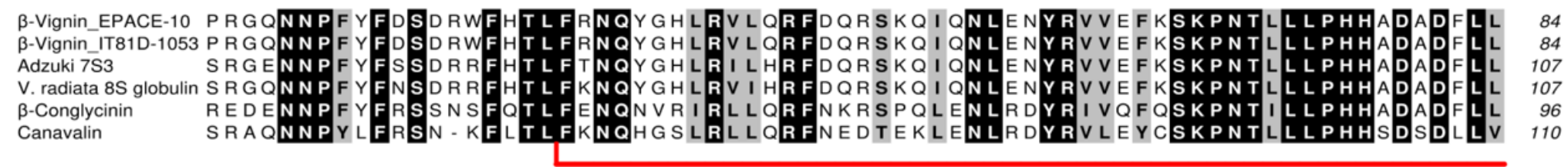

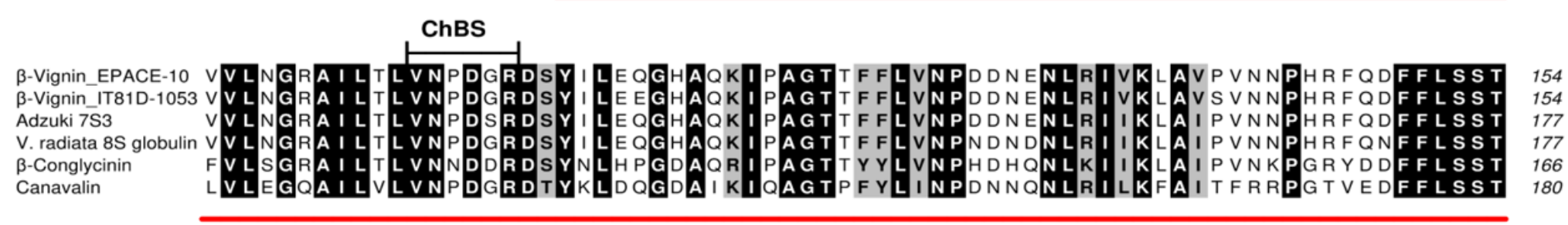

Cupin_1 domain

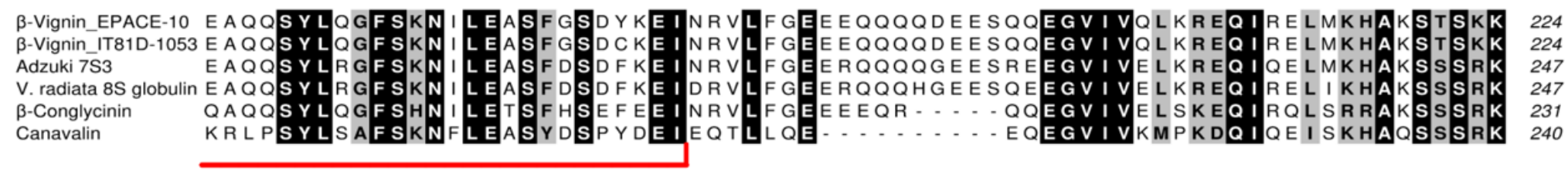

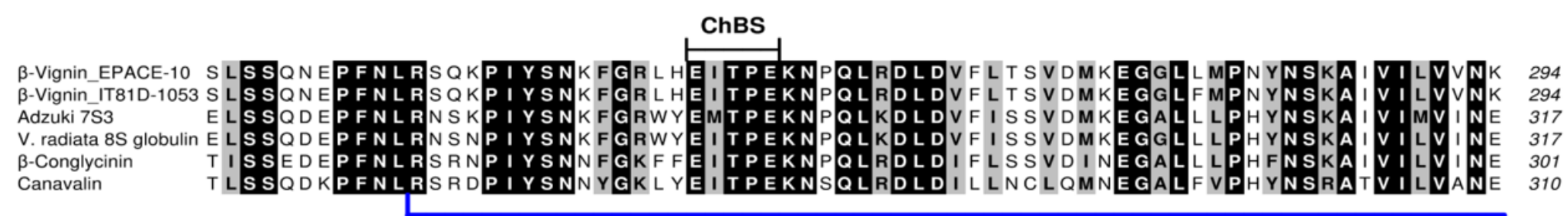

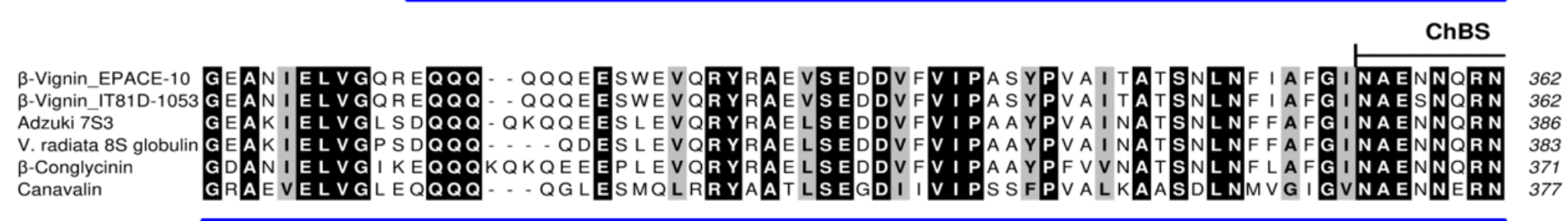
ChBS Cupin_1 domain B-Vignin_EPACE-10 FLAGEEGNVMSETPTEALDVTFPASGEKVEKLINKQSDSHFTDAQPEQQQREEDRKGRKGPLSS ILGTFY 432 B-Vignin_IT81D-1053 F L AGEEDNVMSE P TEVLDVTFPASGEKVEKLINKGSDSHFTDAQPEQQQREEDRKGRKGPLSS ILDSLY 432

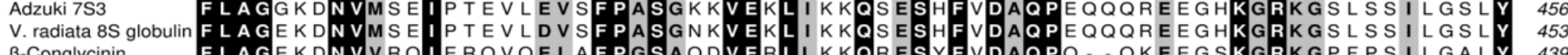

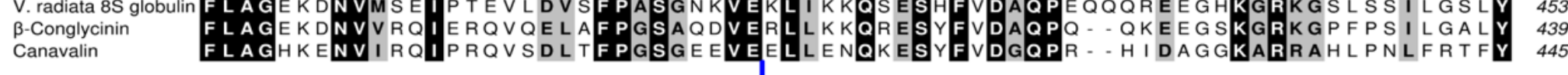


Fig. S1. Multiple sequence alignment of the amino acid sequences of $\beta$-vignin with the primary structures of representative vicilin-like 7S globulins. Amino acid sequences of $\beta$-vignin obtained from V. unguiculata genotypes EPACE-10 (sequence S2) and IT81D-1053 (sequence R2) were aligned with those of V. angularis (adzuki bean) 7S globulin-3 (Adzuki 7S3;UniProtKB accession number: A0A0S3SX36), V. radiata 8S globulin (UniProtKB accession number: Q198W3), $\beta$-conglycinin (from Glycine max; UniProtKB accession number: P25974) and canavalin (from Canavalia ensiformis; UniProtKB accession number: P50477). Segments in the primary structures of $\beta$-vignin that were shown to contribute to their chitin-binding site (ChBS), as evidenced by computational simulations, are indicated. The alignment was edited using the program ALINE.

Table S2- the 77 cupins sequences of the $V$. unguiculata showed the absence or presence of signal peptides and positions of cleavage sites. Signal peptides

\# Measure Position $\max$. C $27 \quad 0.110$

$\max . \mathrm{Y} \quad 27 \quad 0.109$

$\max$. S $22 \quad 0.134$

mean $S \quad 1-26 \quad 0.108$

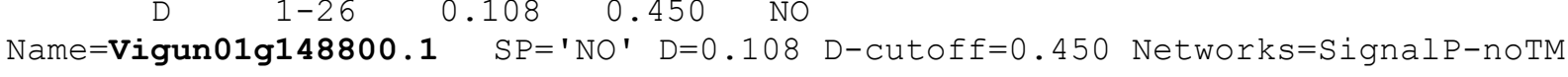

\# Measure Position Value Cutoff signal peptide?

$\max . \mathrm{C} 22 \quad 0.439$

$\max . \mathrm{Y} 22 \quad 0.637$

$\max . \mathrm{S} \quad 12 \quad 0.974$

mean S $1-21 \quad 0.922$

$\begin{array}{lllll}D & 1-21 & 0.791 & 0.450 & \text { YES }\end{array}$

Iigun01g205600.1 SP='YES' Cleavage site between pos. 21 and 22: ITA-SD D=0.791 D-cutoff=0.450

Networks=SignalP-noTM

$\begin{array}{lcc}\text { \# Measure } & \text { Position } & \text { Value } \\ \text { max. C } & 22 & 0.712 \\ \max . \mathrm{Y} & 22 & 0.790 \\ \max . \mathrm{S} & 13 & 0.959\end{array}$

Cutoff signal peptide?

$\max . \mathrm{Y} 22 \quad 0.790$ 


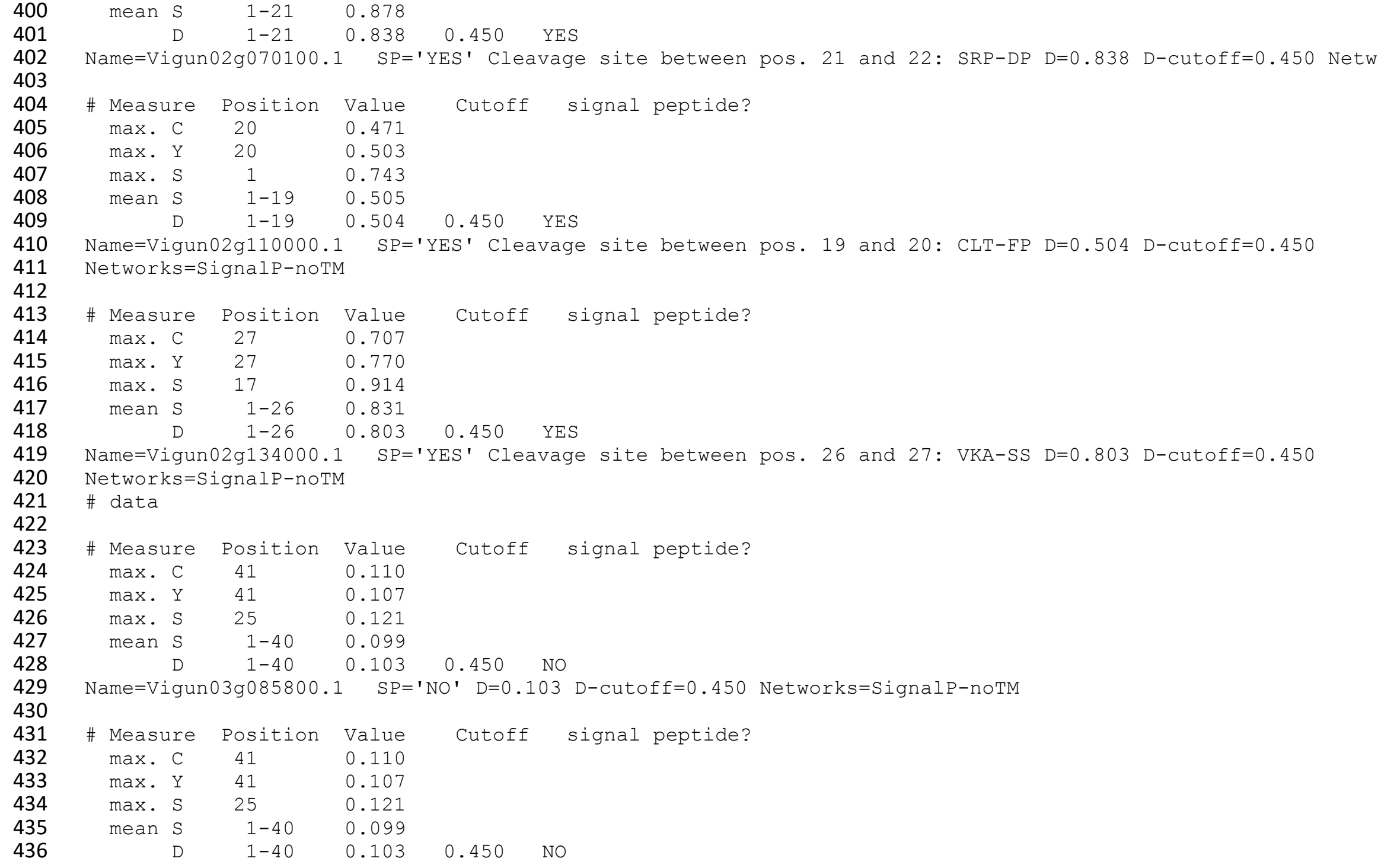


437

438

439

440

441

442

443

444

445

446

447

448

449

450

451

452

453

454

455

456

457

458

459

460

461

462

463

464
465

465

466

467

468

469

470

471

472

473

Name=Vigun03g085800.2 SP='NO' $\mathrm{D}=0.103$ D-cutoff=0.450 Networks=SignalP-noTM

$\begin{array}{lccccc}\text { \# Measure } & \text { Position } & \text { Value } & \text { Cutoff } & \text { signal peptide? } \\ \max . \text { C } & 13 & 0.123 & & \\ \max . \text { Y } & 58 & 0.107 & & \\ \max . \text { S } & 13 & 0.140 & & \\ \text { mean S } & 1-57 & 0.101 & & \\ \text { D } & 1-57 & 0.104 & 0.450 & \text { NO }\end{array}$

Name=Vigun03g085900.1 SP='NO' $\mathrm{D}=0.104$ D-cutoff=0.450 Networks=SignalP-noTM

\# Measure Position Value Cutoff signal peptide?

$\max . \mathrm{C} \quad 13 \quad 0.123$

$\max . \mathrm{Y} \quad 58 \quad 0.107$

$\max \begin{array}{lll}\mathrm{S} & 13 & 0.140\end{array}$

mean $S \quad 1-57 \quad 0.101$

Name=Vigun03g085900.2 SP='NO' $\mathrm{D}=0.104$ D-cutoff=0.450 Networks=SignalP-noTM

$\begin{array}{lccccc}\text { \# Measure } & \text { Position } & \text { Value } & \text { Cutoff } & \text { signal peptide? } \\ \max . & \text { C } & 24 & 0.140 & & \\ \max . \text { Y } & 12 & 0.159 & & \\ \text { max. S } & 1 & 0.327 & & \\ \text { mean S } & 1-11 & 0.217 & & \\ & \text { D } & 1-11 & 0.182 & 0.500 & \text { NO }\end{array}$

Name=Vigun03g254300.1 SP='NO' D=0.182 D-cutoff=0.500 Networks=SignalP-TM

\# Measure Position Value Cutoff signal peptide?

$\max . \mathrm{C} 20 \quad 0.835$

$\max . \mathrm{Y} \quad 20 \quad 0.862$

$\max . \mathrm{S} \quad 12 \quad 0.935$

mean S $1-19 \quad 0.891$

Name=Vigun03g297500.1 SP='YES' Cleavage site between pos. 19 and 20: AFS-EE D=0.877 D-Cutoff=0.450 Networks=Signal $\mathrm{P}-\mathrm{noTM}$

\# Measure Position Value Cutoff signal peptide? $\max . \mathrm{C} \quad 48 \quad 0.127$ 


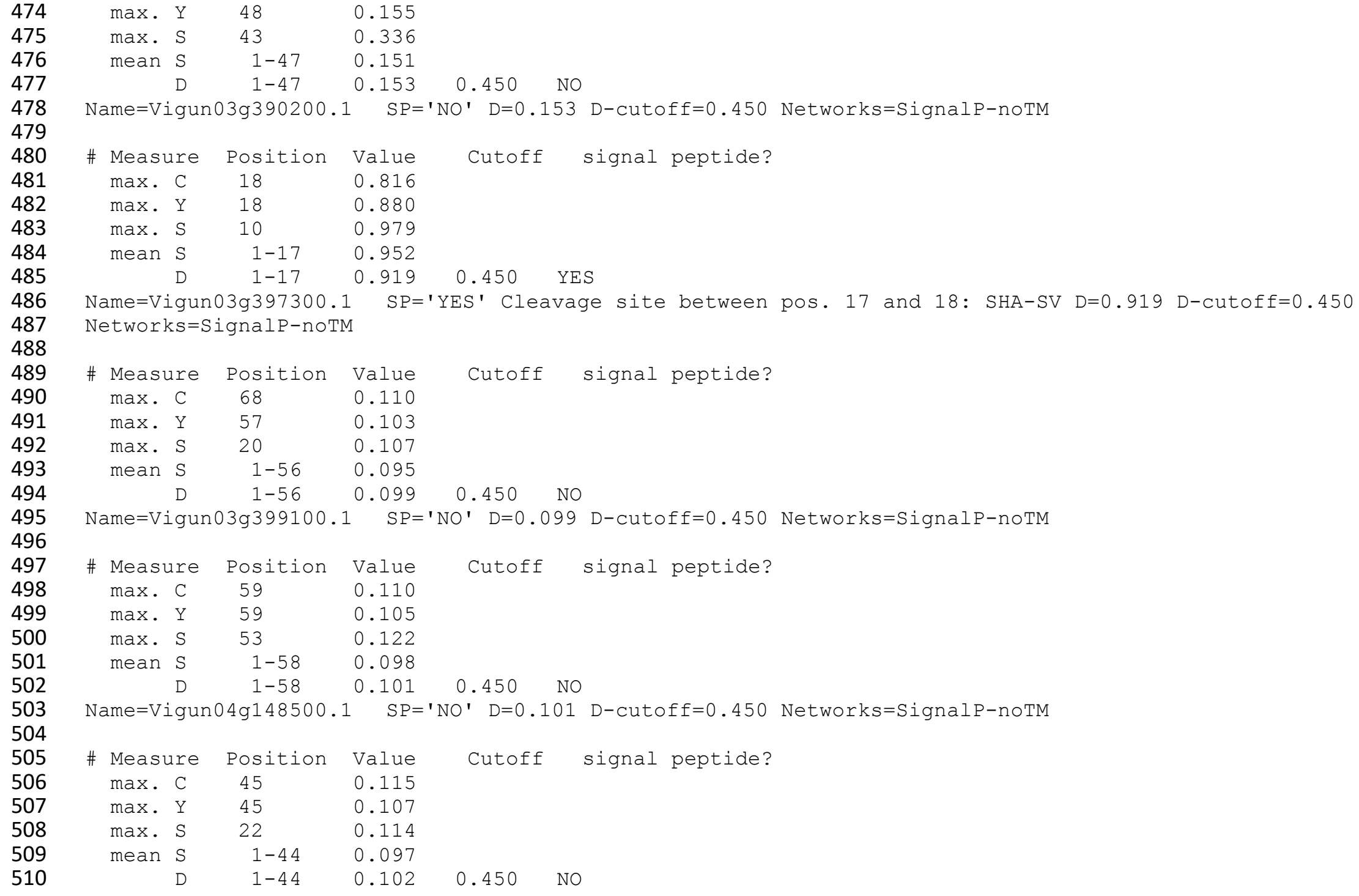

496

497 
511

512

513

514

515

516

517

518

519

520

521

522

523

524

525

526

527

528

529

530

531

532

533

534

535

536

537

538

539

540

541

542

543

544

545

547

Name=Vigun04g167800.1 SP='NO' $\mathrm{D}=0.102$ D-cutoff=0.450 Networks=SignalP-noTM

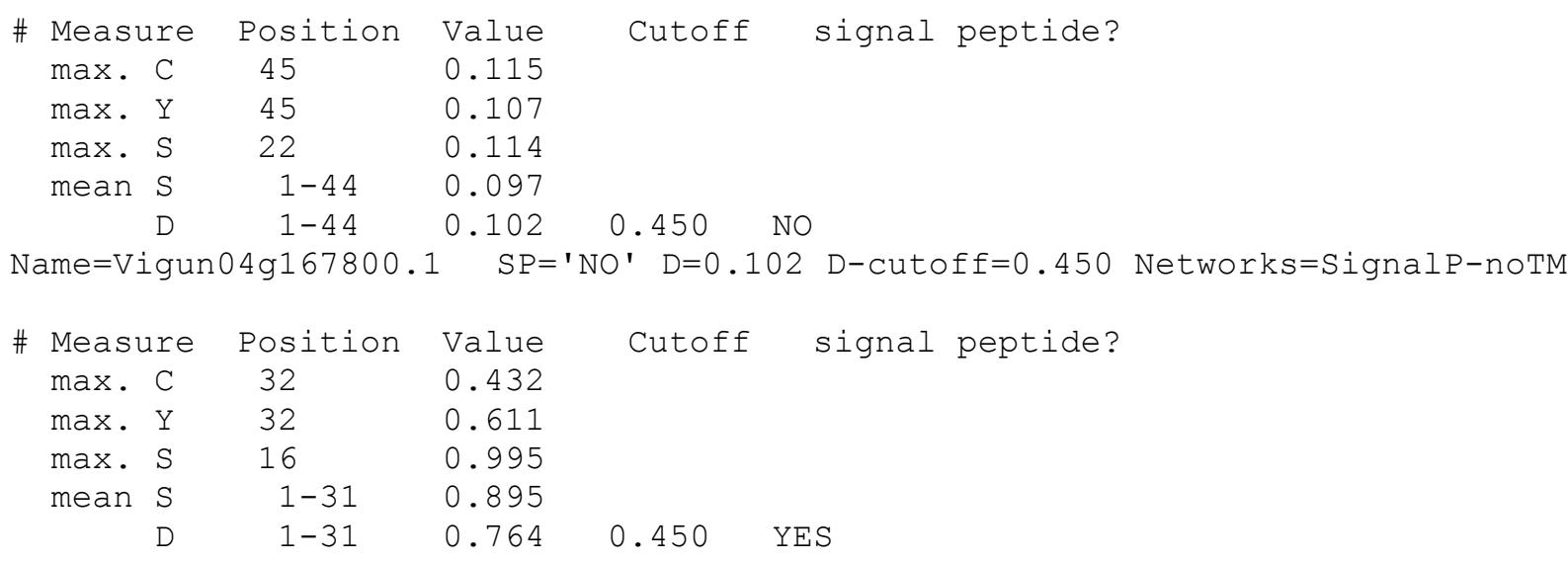


ame=Vigun $05 \mathrm{~g} 166300.1$
0.818
$0.450 \quad Y E S$

Networks=SignalP-noTM

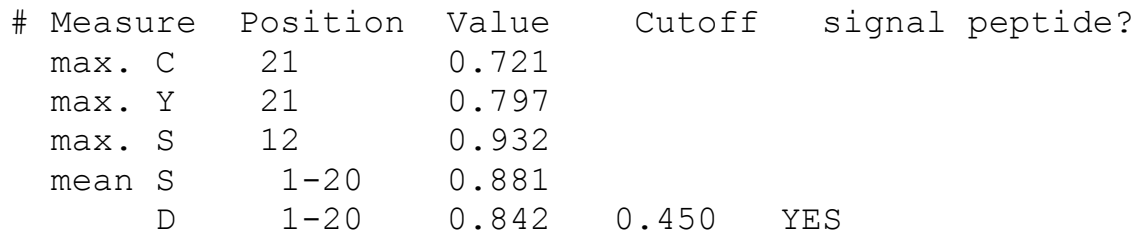

Name=Vigun05g166800.1 SP='YES' Cleavage site between pos. 20 and 21: ASA-YD D=0.842 D-Cutoff=0.450 Networks=SignalP-noTM

$\begin{array}{lcccc}\text { \# } \text { Measure } & \text { Position } & \text { Value } & \text { Cutoff } & \text { signal peptide? } \\ \max . \text { C } & 21 & 0.721 & & \\ \max . \text { Y } & 21 & 0.797 & & \\ \max . \text { S } & 12 & 0.932 & & \\ \text { mean S } & 1-20 & 0.881 & & \\ \text { D } & 1-20 & 0.842 & 0.450 & \text { YES }\end{array}$

Name=Vigun05g166900.1 SP='YES' Cleavage site between pos. 20 and 21: ASA-YD D=0.842 D-Cutoff=0.450 Networks=Signal $\mathrm{P}-$ noTM

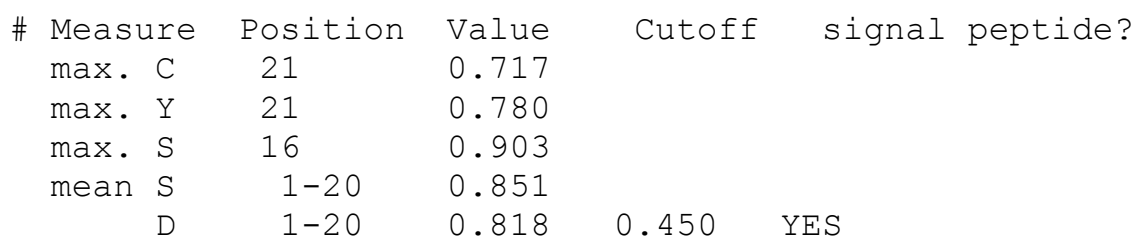

$\begin{array}{lcc}\text { \# Measure } & \text { Position } & \text { Value } \\ \max . \mathrm{C} & 26 & 0.408 \\ \max . \mathrm{Y} & 26 & 0.560 \\ \max . \mathrm{S} & 17 & 0.859\end{array}$

Cutof signal peptide? 
Networks=SignalP-noTM

$\begin{array}{lcccc}\text { \# Measure } & \text { Position } & \text { Value } & \text { Cutoff } & \text { signal peptide? } \\ \text { max. C } & 24 & 0.547 & & \\ \text { max. Y } & 20 & 0.703 & & \\ \text { max. S } & 3 & 0.961 & & \\ \text { mean S } & 1-19 & 0.920 & & \\ \text { D } & 1-19 & 0.820 & 0.450 & \text { YES }\end{array}$

Name=Vigun05g250800.1 SP='YES' Cleavage site between pos. 19 and 20: GVA-VT D=0.820 D-Cutoff=0.450 Networks=SignalP-noTM

$\begin{array}{lcccc}\text { \# Measure } & \text { Position } & \text { Value } & \text { Cutoff } & \text { signal peptide? } \\ \max . \text { C } & 20 & 0.561 & & \\ \max . \text { Y } & 20 & 0.721 & & \\ \text { max. S } & 3 & 0.964 & & \\ \text { mean S } & 1-19 & 0.926 & & \\ \text { D } & 1-19 & 0.832 & 0.450 & \text { YES }\end{array}$

Name=Vigun05g250900.1 SP='YES' Cleavage site between pos. 19 and 20: GVA-VT D=0.832 D-Cutoff=0.450 Networks=SignalP-noTM

608

608

\# Measure Position $\max . \mathrm{C} 23 \quad 0.229$ $\max . \mathrm{Y} 23 \quad 0.216$

$\begin{array}{lll}\max . \mathrm{S} & 3 & 0.291\end{array}$

mean S $1-22 \quad 0.204$

$\begin{array}{lllll}\text { D } & 1-22 & 0.210 & 0.450 & \text { NO }\end{array}$

Name=Vigun05g251000.1 SP='NO' $\mathrm{D}=0.210$ D-cutoff=0.450 Networks=SignalP-noTM

614

615

616

\section{Value}

$\max C \quad 390.109$

$\max . \mathrm{Y} \quad 12 \quad 0.108$

$\max . \mathrm{S} \quad 1 \quad 0.131$

mean S $1-11 \quad 0.094$

620 mean S 
632

633

634

635

636

637

638

639

640

641

642

643

644

645

646

647

648

649

650

651

652

653

654

655

656

657

658

Name=Vigun05g251000.2 SP='NO' $\mathrm{D}=0.101$ D-cutoff=0.450 Networks=SignalP-noTM

$\begin{array}{lcccc}\text { \# Measure } & \text { Position } & \text { Value } & \text { Cutoff } & \text { signal peptide? } \\ \text { max. C } & 16 & 0.129 & & \\ \text { max. Y } & 37 & 0.105 & & \\ \text { max. S } & 25 & 0.111 & & \\ \text { mean S } & 1-36 & 0.093 & & \\ \text { D } & 1-36 & 0.099 & 0.450 & \text { NO }\end{array}$

Name=Vigun05g254700.1 SP='NO' $\mathrm{D}=0.099$ D-cutoff=0.450 Networks=SignalP-noTM

\# Measure Position Value Cutoff signal peptide? $\max . \mathrm{C} 23 \quad 0.109$

$\max . \mathrm{Y} \quad 11 \quad 0.138$

$\max \mathrm{S} \quad 2 \quad 0.243$

mean $S \quad 1-10 \quad 0.144$

Name=Vigun06g057200.1 SP='NO' $\mathrm{D}=0.141$ D-cutoff=0.450 Networks=SignalP-noTM

$\begin{array}{lccccc}\text { \# Measure } & \text { Position } & \text { Value } & \text { Cutoff } & \text { signal peptide? } \\ \text { max. C } & 66 & 0.110 & & \\ \max . \text { Y } & 11 & 0.119 & & \\ \text { max. S } & 2 & 0.205 & & \\ \text { mean S } & 1-10 & 0.128 & & \\ \text { D } & 1-10 & 0.124 & 0.450 & \text { NO }\end{array}$

Name=Vigun06g110700.1 SP='NO' D=0.124 D-cutoff=0.450 Networks=SignalP-noTM

\# Measure Position Value Cutoff signal peptide?

$\max . \mathrm{C} 23 \quad 0.717$

$\max . \mathrm{Y} 23 \quad 0.833$

$\max . \mathrm{S} \quad 16 \quad 0.994$

mean $S \quad 1-22 \quad 0.967$

$0.905 \quad 0.450 \quad Y E S$

Name=Vigun06g138000.1 SP='YES' Cleavage site between pos. 22 and 23: AAA-TS D=0.905 D-Cutoff=0.450 Networks=Signal - -notM

\# Measure Position Value Cutoff signal peptide? $\max$ C $21 \quad 0.870$ 


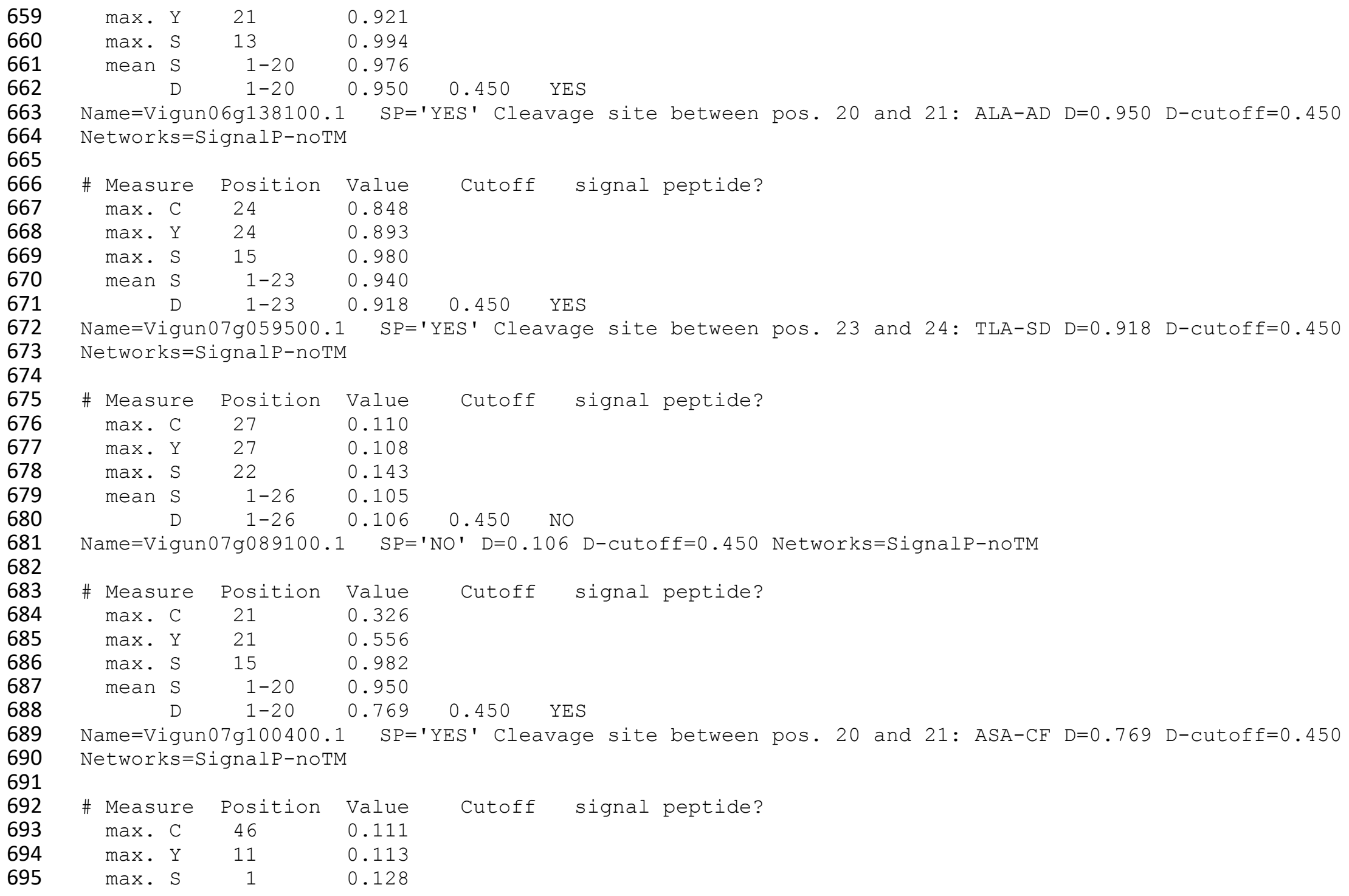




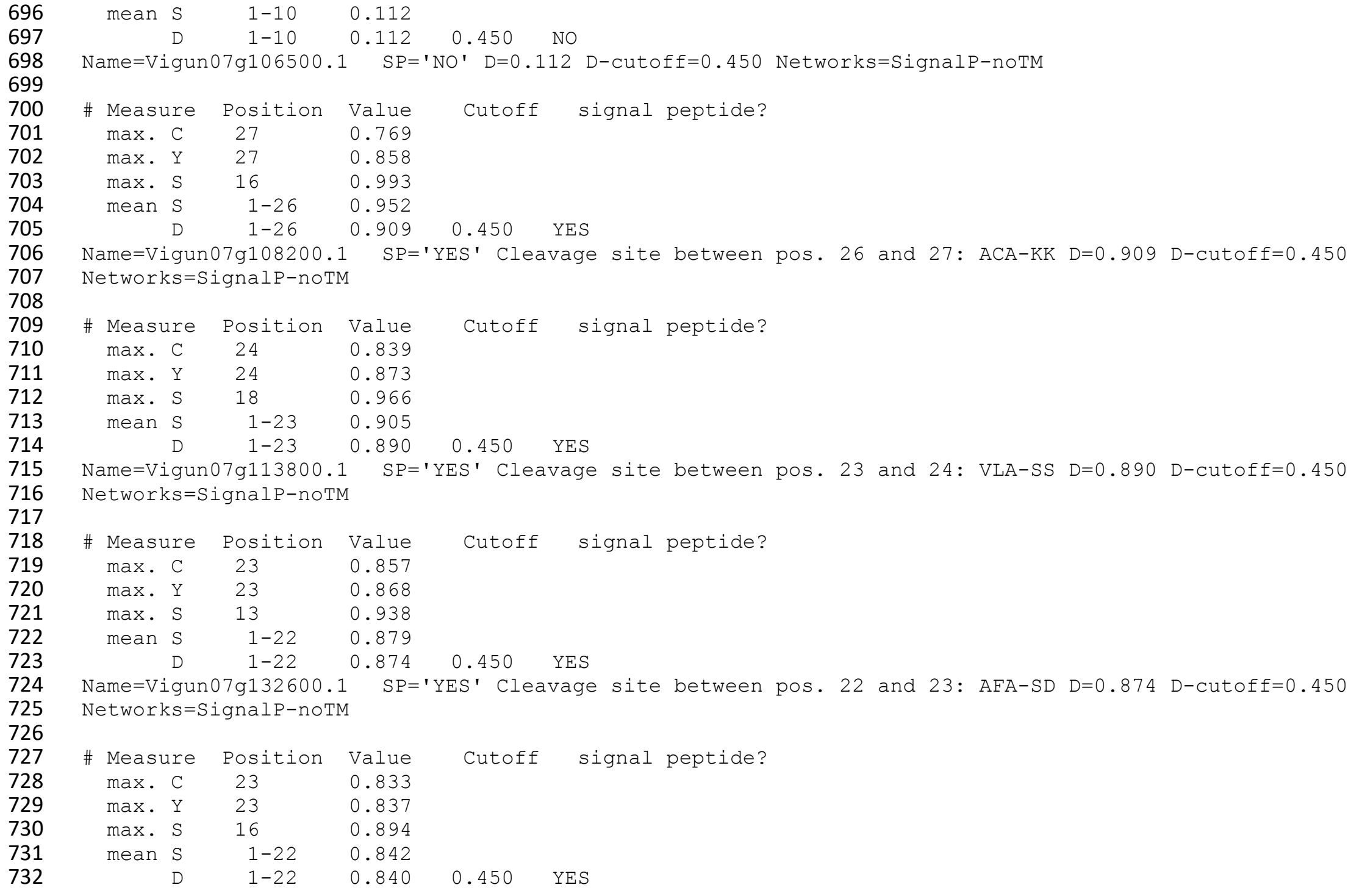


733

Name=Vigun07g132700.1

SP='YES' Cleavage site between pos. 22 and 23: AFA-SD D=0.840 D-cutoff=0.450 Networks=SignalP-noTM

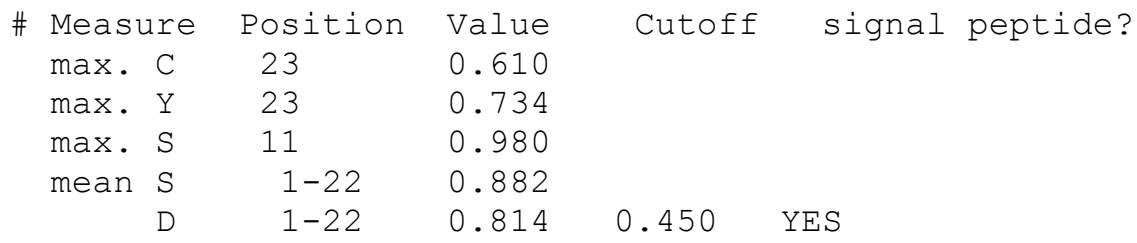

Name=Vigun07g133900.1 SP='YES' Cleavage site between pos. 22 and 23: VSS-DP D=0.814 D-Cutoff=0.450 Networks=SignalP-noTM

$\begin{array}{lcccc}\text { \# Measure } & \text { Position } & \text { Value } & \text { Cutoff } & \text { signal peptide? } \\ \text { max. C } & 49 & 0.112 & & \\ \text { max. Y } & 49 & 0.108 & & \\ \text { max. S } & 38 & 0.117 & & \\ \text { mean S } & 1-48 & 0.100 & & \\ \text { D } & 1-48 & 0.104 & 0.450 & \text { NO }\end{array}$

Name=Vigun07g159700.1 SP='NO' D=0.104 D-cutoff=0.450 Networks=SignalP-noTM

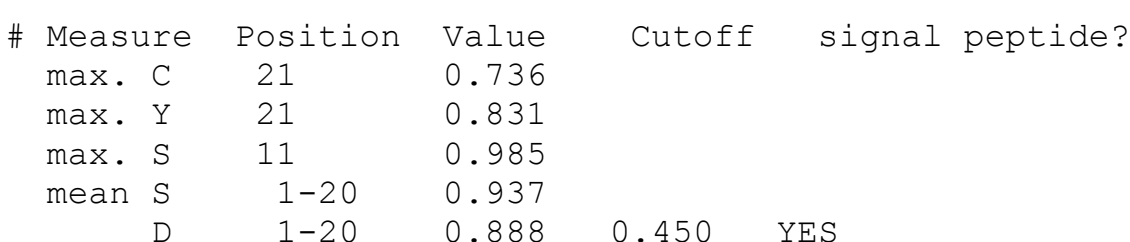

Name=Vigun07g160600.1 SP='YES' Cleavage site between pos. 20 and 21: AFA-YD D=0.888 D-Cutoff=0.450 Networks=SignalP-noTM

$\begin{array}{lcccc}\text { \# Measure } & \text { Position } & \text { Value } & \text { Cutoff } & \text { signal peptide? } \\ \max . \text { C } & 21 & 0.735 & & \\ \max . \mathrm{Y} & 21 & 0.830 & & \\ \max . \mathrm{S} & 11 & 0.985 & & \\ \text { mean S } & 1-20 & 0.936 & & \\ \text { D } & 1-20 & 0.887 & 0.450 & \text { YES }\end{array}$

Name=Vigun07g160600.2 SP='YES' Cleavage site between pos. 20 and 21: AFA-YD D=0.887 D-Cutoff=0.450 Networks=SignalP-noTM 
805 


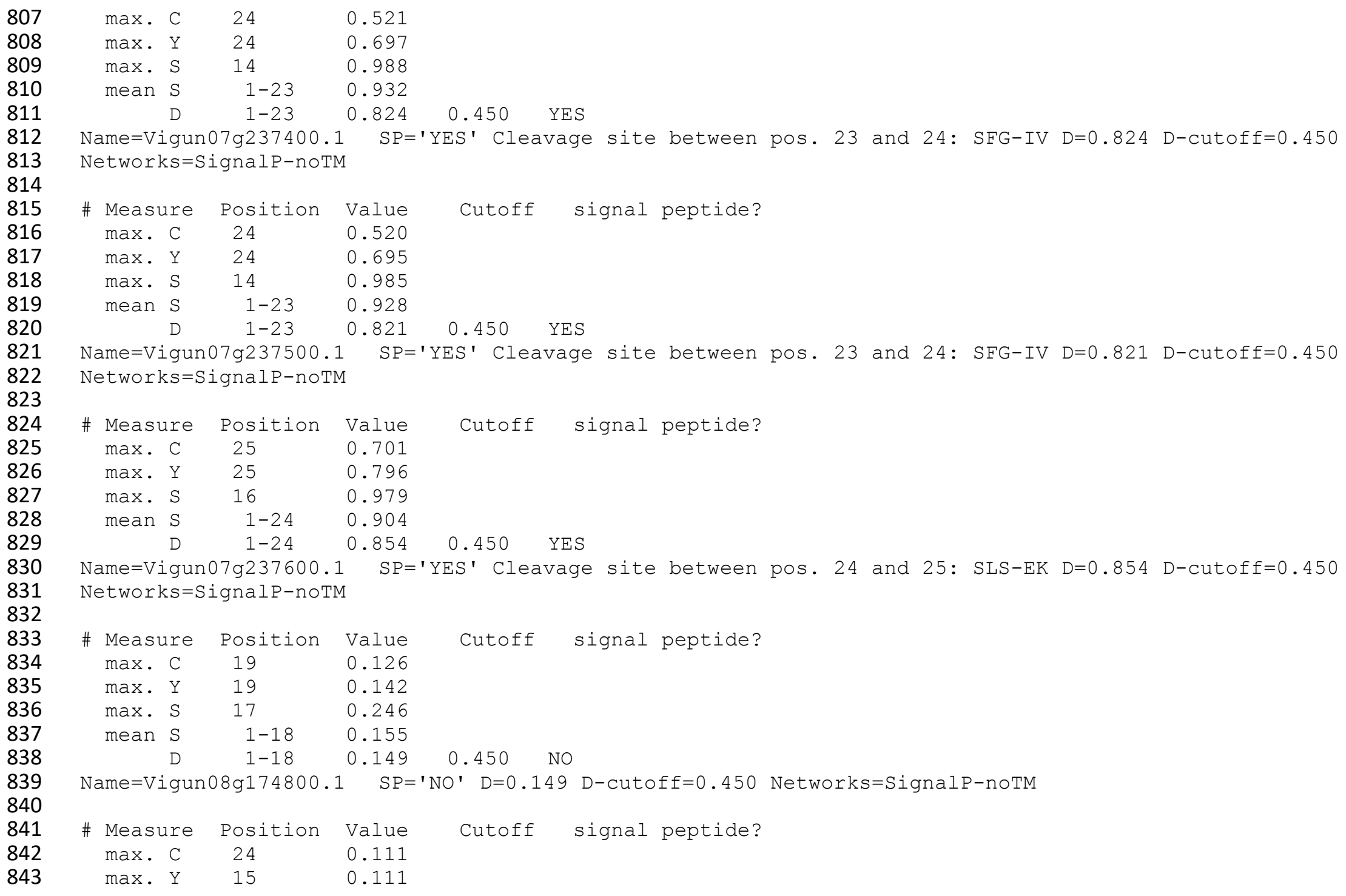




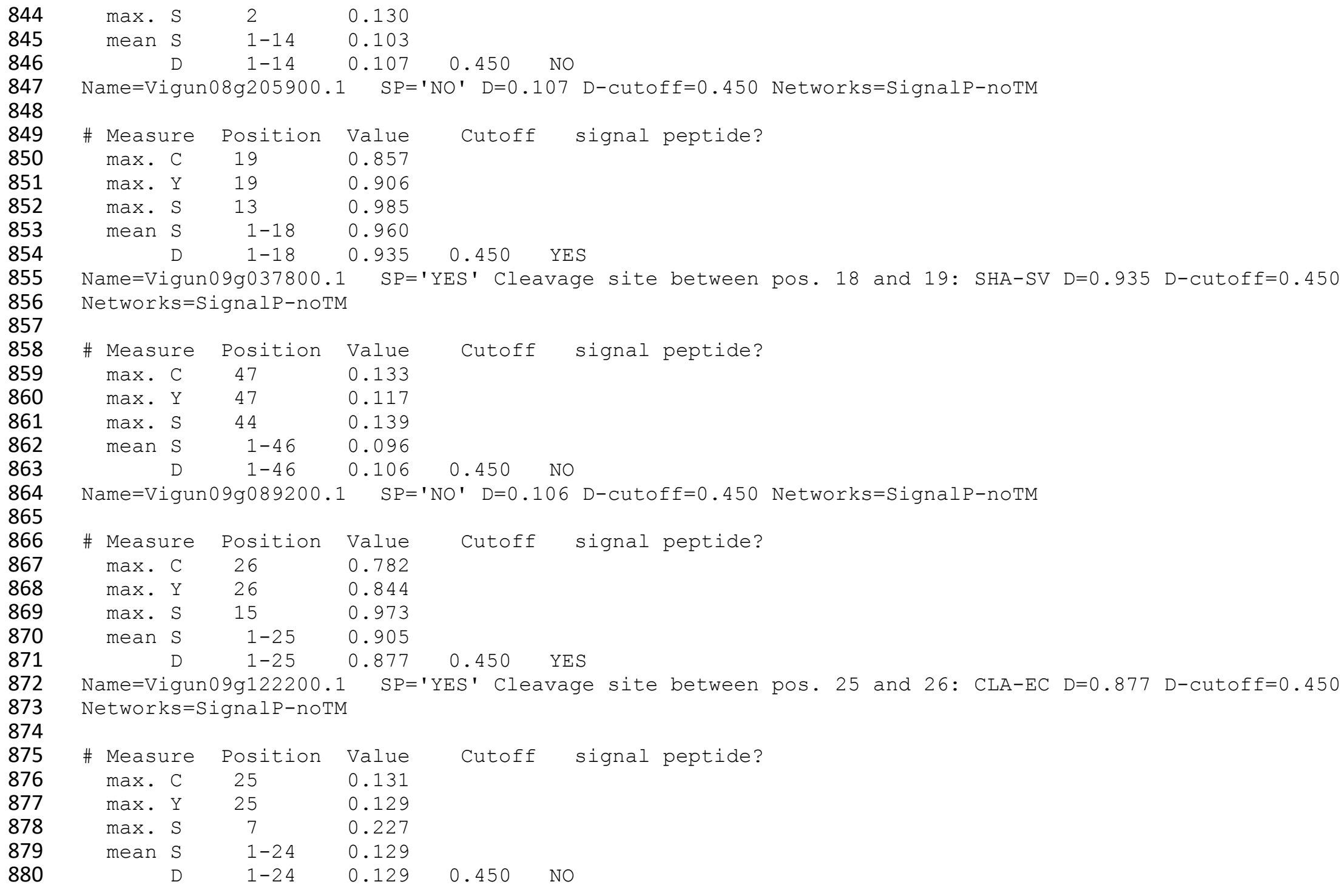


891

892

Name=Vigun09g177900.1 SP='NO' D=0.129 D-cutoff=0.450 Networks=SignalP-noTM

$\begin{array}{lcccc}\text { \# Measure } & \text { Position } & \text { Value } & \text { Cutoff } & \text { signal peptide? } \\ \max . \text { C } & 24 & 0.511 & & \\ \max . \text { Y } & 24 & 0.689 & & \\ \max . \text { S } & 15 & 0.986 & & \\ \text { mean S } & 1-23 & 0.927 & & \\ \text { D } & 1-23 & 0.817 & 0.450 & \text { YES }\end{array}$

Name=Vigunlog081000.1 SP='YES' Cleavage site between pos. 23 and 24: SFG-IV D=0.817 D-Cutoff=0.450 Networks=SignalP-noTM

$\begin{array}{llllll}\text { \# Measure } & \text { Position } & \text { Value } & \text { Cutoff } & \text { signal peptide? } \\ \max . & \text { C } & 21 & 0.828 & & \\ \max . \text { Y } & 21 & 0.868 & & \\ \text { max. S } & 11 & 0.951 & & \\ \text { mean S } & 1-20 & 0.908 & & \\ & \text { D } & 1-20 & 0.889 & 0.450 & \text { YES }\end{array}$

Name=Vigunlog086400.1 SP='YES' Cleavage site between pos. 20 and 21: VSA-YD D=0.889 D-Cutoff=0.450 Networks=SignalP-noTM

$\begin{array}{lcccc}\text { \# Measure } & \text { Position } & \text { Value } & \text { Cutoff } & \text { signal peptide? } \\ \max . \mathrm{C} & 21 & 0.816 & & \\ \max . \mathrm{Y} & 21 & 0.852 & & \\ \max . \mathrm{S} & 11 & 0.944 & & \\ \text { mean S } & 1-20 & 0.887 & & \\ \text { D } & 1-20 & 0.871 & 0.450 & \text { YES }\end{array}$

Name=Vigunlog086500.1 SP='YES' Cleavage site between pos. 20 and 21: VSA-YD D=0.871 D-Cutoff=0.450 Networks=Signal $\mathrm{P}-$ noTM

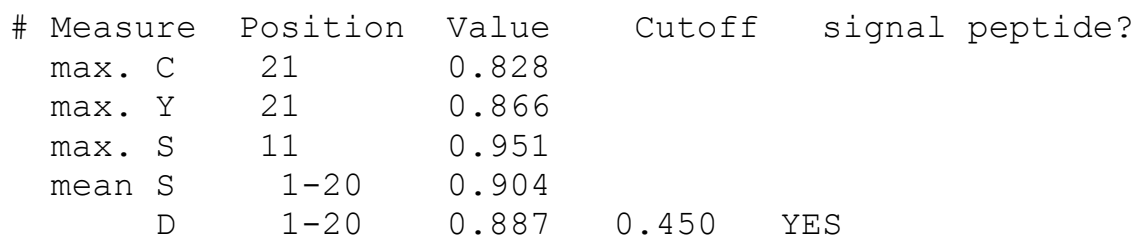

Name=Vigun10g086600.1 SP='YES' Cleavage site between pos. 20 and 21: VSA-YD D=0.887 D-Cutoff=0.450 Networks=SignalP-noTM 
918

919

920

921

922

923

924

925

926

927

928

929

930

931

932

933

934

935

936

937

938

939

940

941

942

943

944

945

946

947

948

949

950

951

952

953

$\begin{array}{lcccc}\text { \# Measure } & \text { Position } & \text { Value } & \text { Cutoff } & \text { signal peptide? } \\ \text { max. C } & 21 & 0.828 & & \\ \max . \text { Y } & 21 & 0.868 & & \\ \text { max. S } & 11 & 0.951 & & \\ \text { mean S } & 1-20 & 0.908 & & \\ \text { D } & 1-20 & 0.889 & 0.450 & \text { YES }\end{array}$

Name=Vigunlog086700.1 SP='YES' Cleavage site between pos. 20 and 21: VSA-YD D=0.889 D-Cutoff=0.450 Networks=SignalP-noTM

$\begin{array}{lcccc}\text { \# Measure } & \text { Position } & \text { Value } & \text { Cutoff } & \text { signal peptide? } \\ \max . \text { C } & 21 & 0.645 & & \\ \max . \text { Y } & 21 & 0.751 & & \\ \max . \text { S } & 11 & 0.931 & & \\ \text { mean S } & 1-20 & 0.870 & & \\ \text { D } & 1-20 & 0.815 & 0.450 & \text { YES }\end{array}$

Name=Vigunlog087300.1 SP='YES' Cleavage site between pos. 20 and 21: VSS-YD D=0.815 D-Cutoff=0.450 Networks=SignalP-noTM

\# Measure Position Value Cutoff signal peptide? max. C $24 \quad 0.533$ $\max . \mathrm{Y} 24 \quad 0.715$

$\max . \mathrm{S} \quad 15 \quad 0.990$

mean S $\quad 1-23 \quad 0.955$

$\begin{array}{lll}1-23 & 0.845 & 0.450 \\ \text { YES }\end{array}$

Name=Vigunlog096400.1 SP='YES' Cleavage site between pos. 23 and 24: SVS-FG D=0.845 D-Cutoff=0.450 Networks=SignalP-noTM

$\begin{array}{lcccc}\text { \# Measure } & \text { Position } & \text { Value } & \text { Cutoff } & \text { signal peptide? } \\ \max . \text { C } & 24 & 0.533 & & \\ \max . \text { Y } & 24 & 0.715 & & \\ \text { max. S } & 15 & 0.990 & & \\ \text { mean S } & 1-23 & 0.955 & & \\ \text { D } & 1-23 & 0.845 & 0.450 & \text { YES }\end{array}$

Name=Vigunlog096600.1 SP='YES' Cleavage site between pos. 23 and 24: SVS-FG D=0.845 D-Cutoff=0.450 Networks=SignalP-noTM 
Name=Vigun10g164300.1 SP='YES' Cleavage site between pos. 20 and 21: SYA-AV D=0.753 D-Cutoff=0.450 Networks=SignalP-noTM

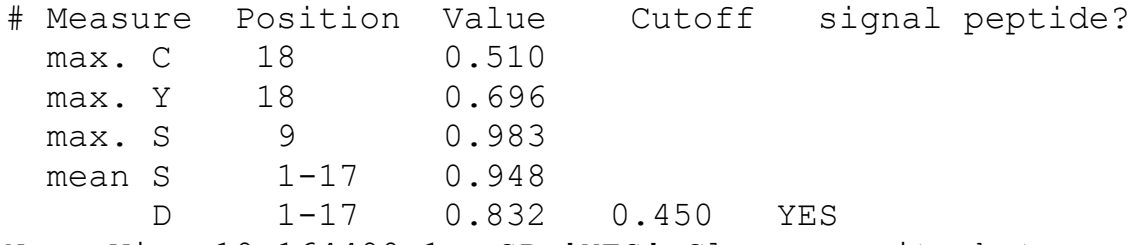




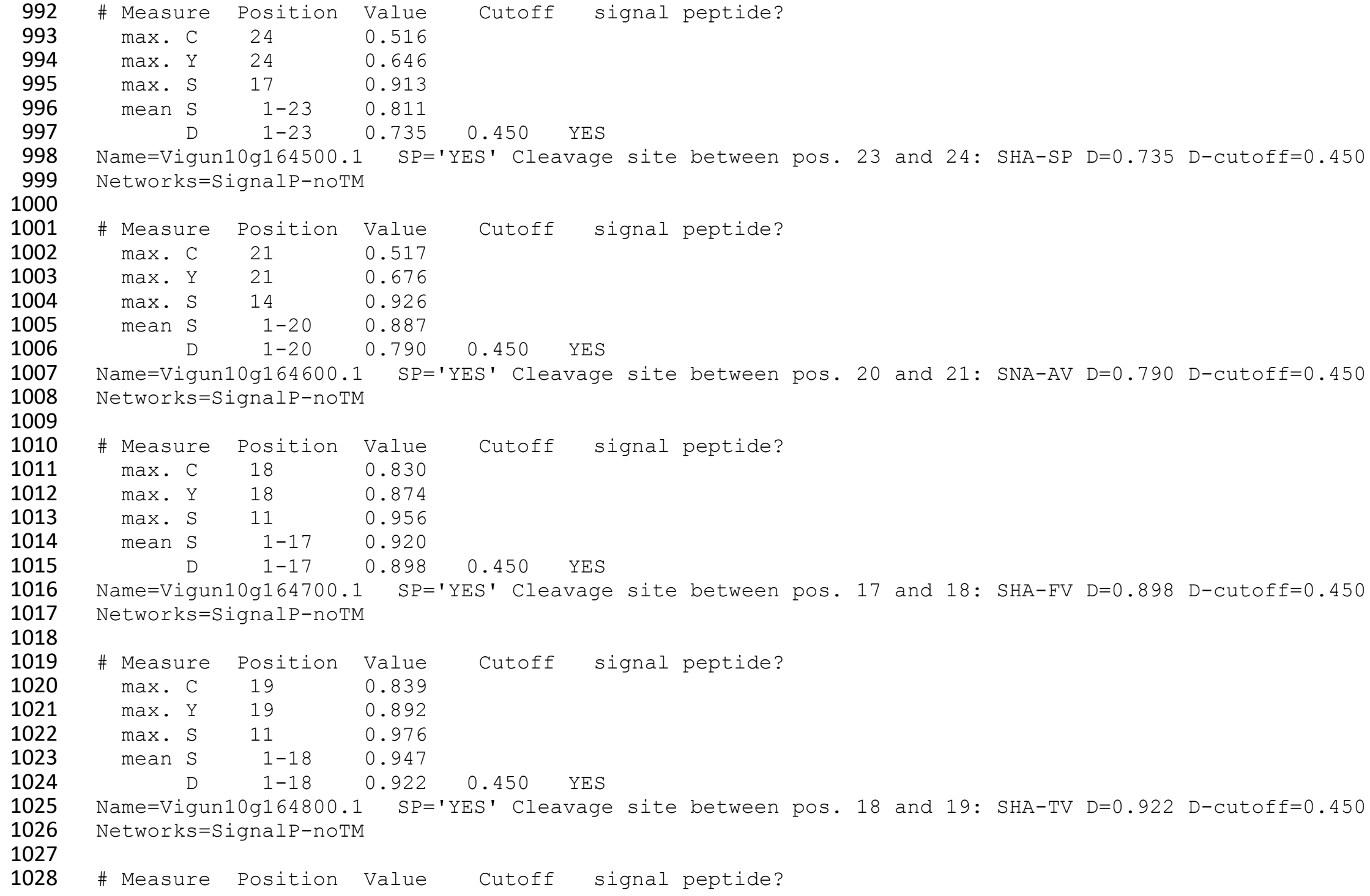




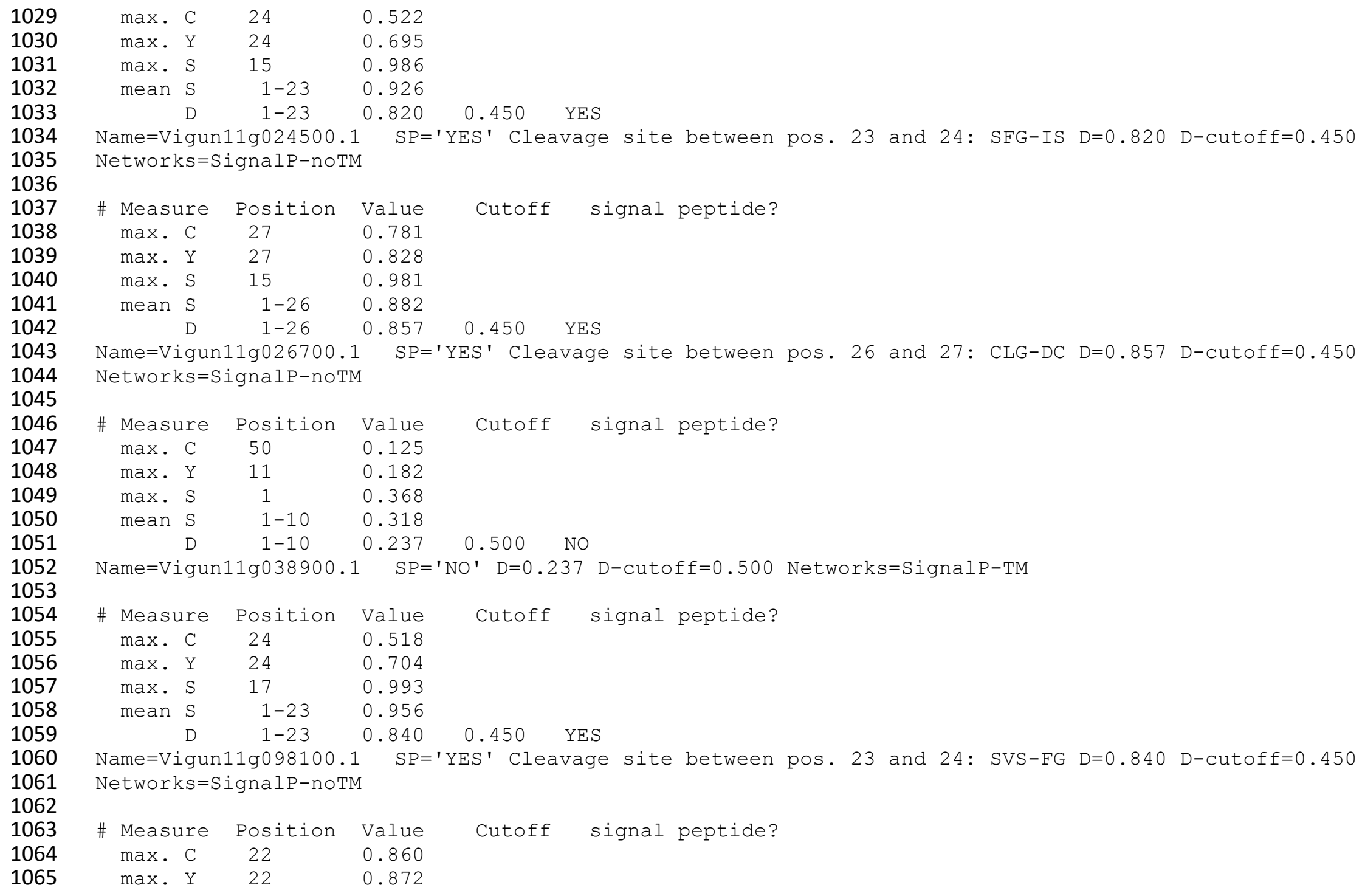




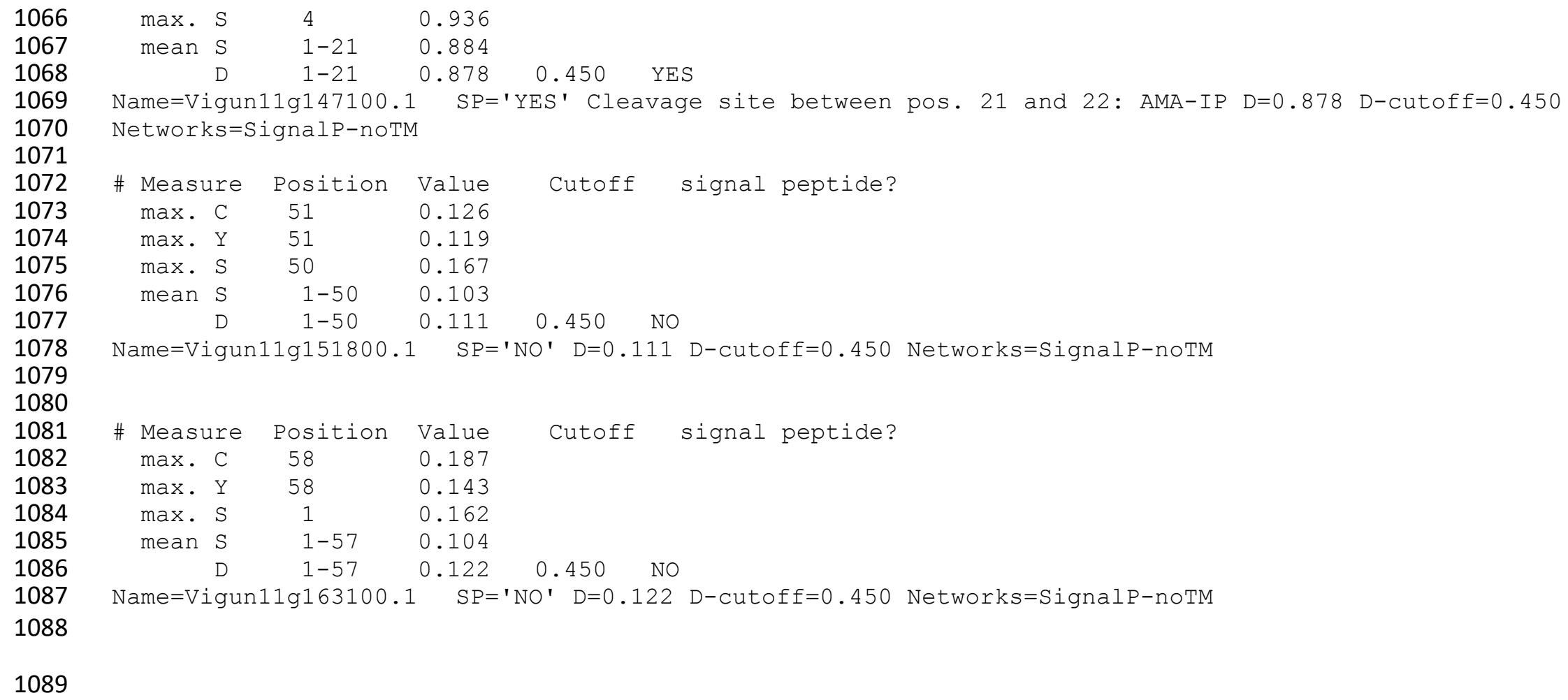

1089

1090 Supplementary material

\begin{tabular}{|c|c|c|c|c|c|c|c|}
\hline Suence Number & Query Name & Hits Found & Identifier & Accession & Clan & Description & Protein size (aa) \\
\hline 1 & Vigun01g148800.1 & 1 & Cupin_3 & PF05899.11 & CL0029 & Protein of unknown function (DUF861) & 110 \\
\hline 2 & Vigun01g205600.1 & 2 & Cupin_1 & PF00190.21 & CL0029 & Cupin & 217 \\
\hline 3 & Vigun02g070100.1 & 2 & Cupin_1 & PF00190.21 & CL0029 & Cupin & 216 \\
\hline 4 & Vigun02g110000.1 & 1 & Cupin_8 & PF13621.5 & CL0029 & Cupin-like domain & 415 \\
\hline 5 & Vigun02g134000.1 & 1 & Cupin_1 & PF00190.21 & CL0029 & Cupin & 631 \\
\hline 6 & Vigun03g085800.1 & 2 & Cupin_1 & PF00190.21 & CL0029 & Cupin & 357 \\
\hline
\end{tabular}




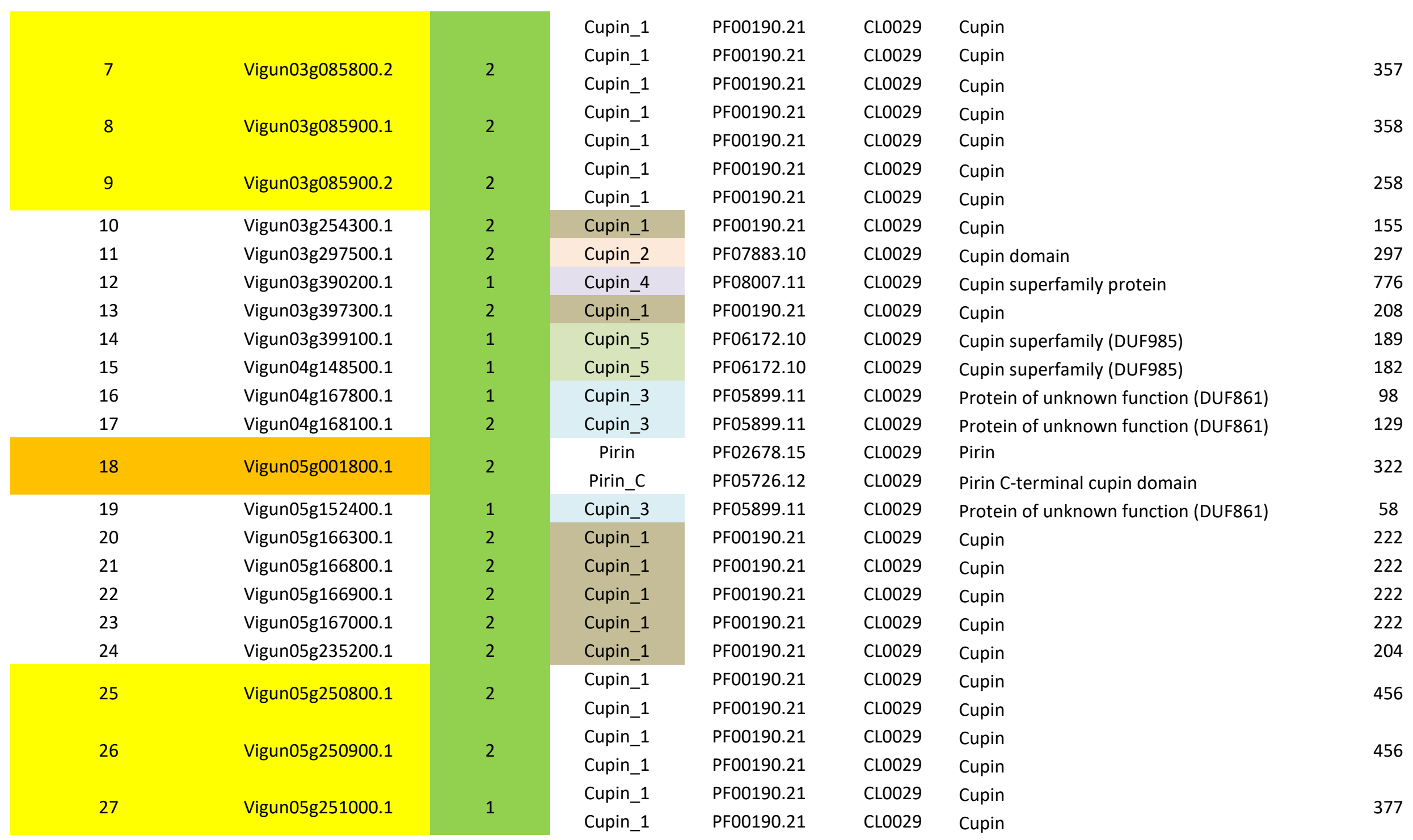




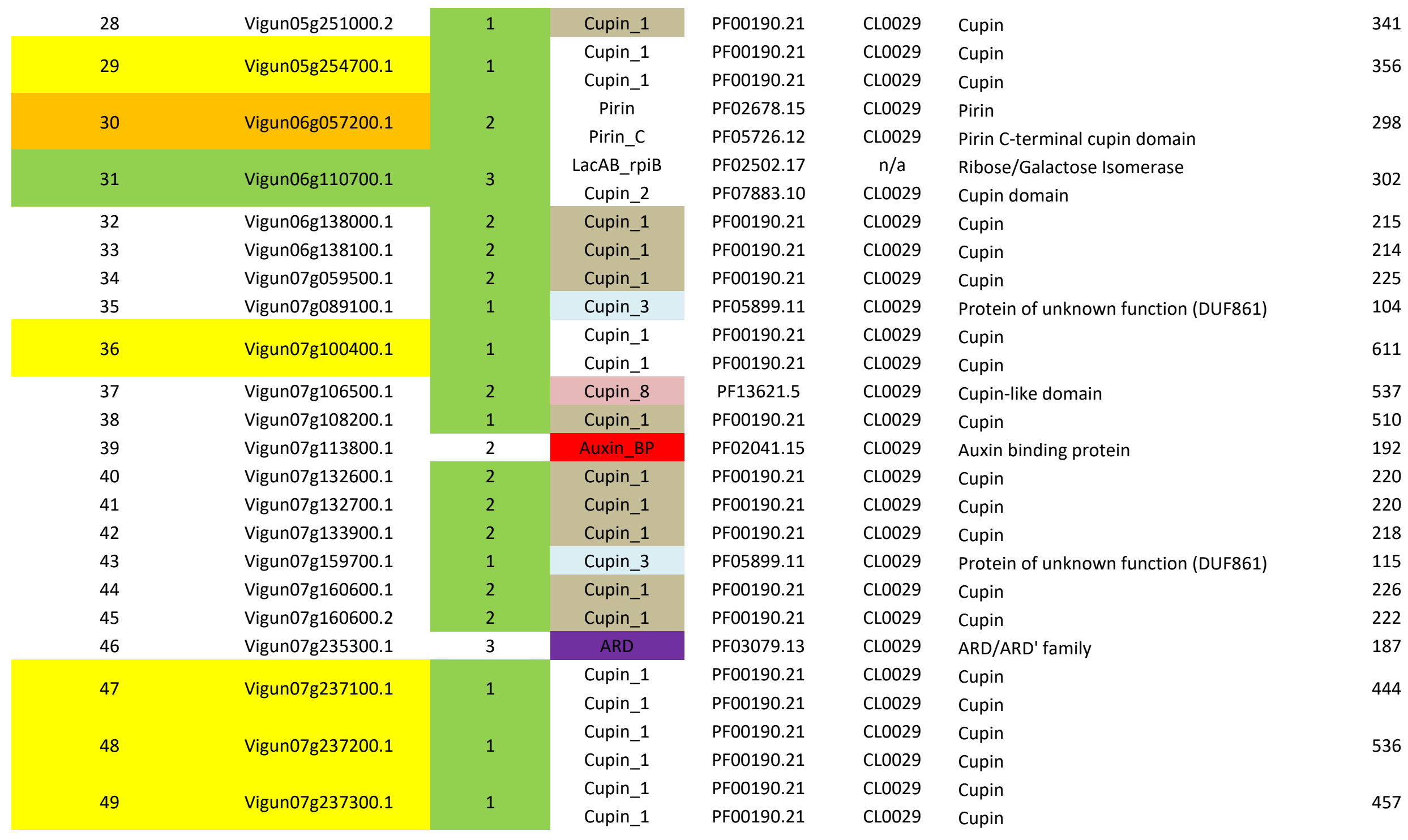




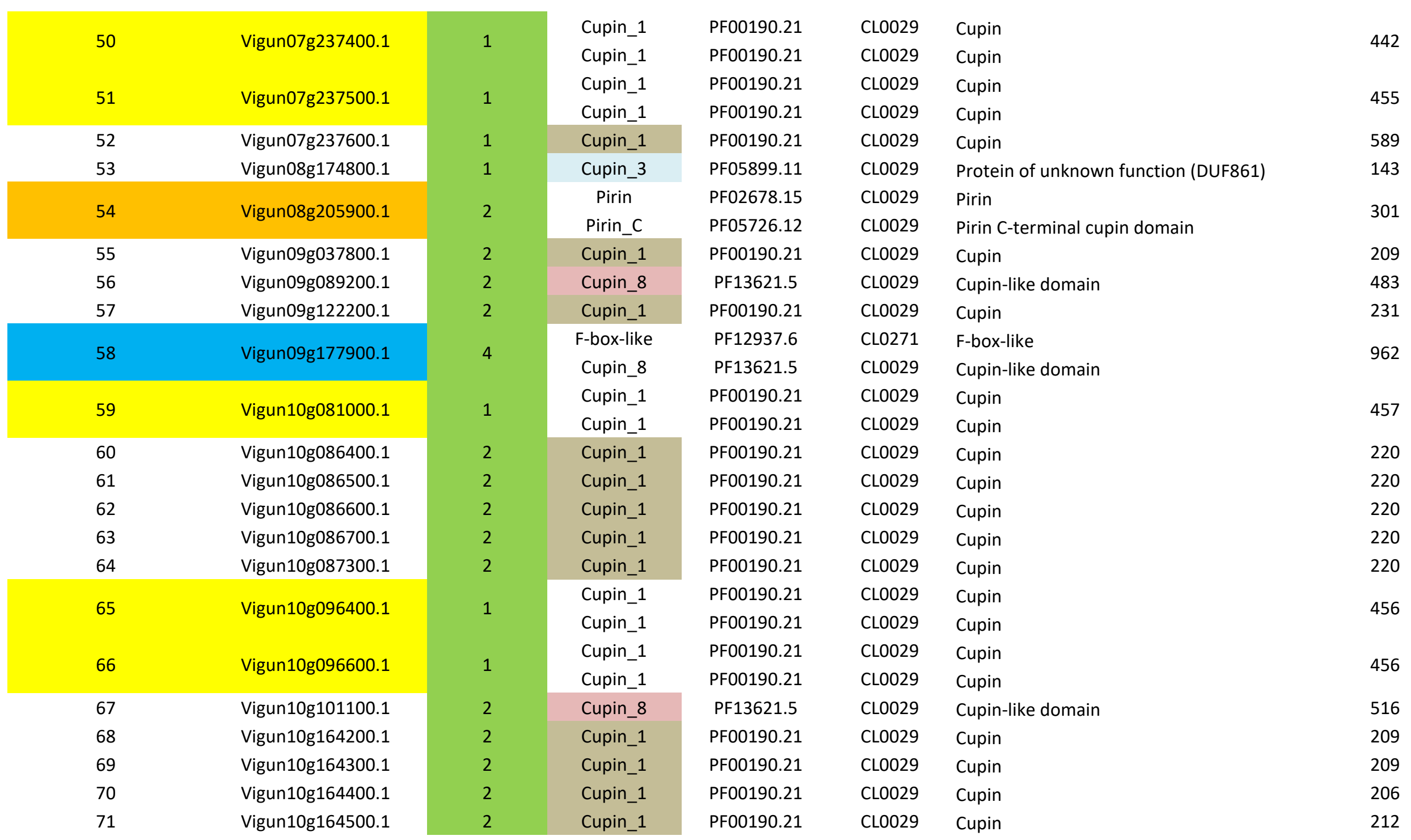




\begin{tabular}{|c|c|c|c|}
\hline 72 & Vigun10g164600.1 & 2 & Cupin_1 \\
\hline 73 & Vigun10g164700.1 & 2 & Cupin_1 \\
\hline 74 & Vigun10g164800.1 & 1 & Cupin_1 \\
\hline \multirow{2}{*}{75} & \multirow{2}{*}{ Vigun11g024500.1 } & \multirow{2}{*}{1} & Cupin_1 \\
\hline & & & Cupin_1 \\
\hline 76 & Vigun11g026700.1 & 2 & Cupin_1 \\
\hline 77 & Vigun11g038900.1 & 1 & Cupin_3 \\
\hline \multirow{2}{*}{78} & \multirow{2}{*}{ Vigun11g098100.1 } & \multirow{2}{*}{2} & Cupin_1 \\
\hline & & & Cupin_1 \\
\hline \multirow{2}{*}{79} & \multirow{2}{*}{ Vigun11g147100.1 } & \multirow{2}{*}{2} & Cupin_1 \\
\hline & & & Cupin_1 \\
\hline \multirow{2}{*}{80} & \multirow{2}{*}{ Vigun11g151800.1 } & \multirow{2}{*}{1} & Cupin_1 \\
\hline & & & Cupin_1 \\
\hline 81 & Vigun11g163100.1 & 3 & Cupin_8 \\
\hline
\end{tabular}

$\begin{array}{lll}\text { PF00190.21 } & \text { CL0029 } & \text { Cupin } \\ \text { PF00190.21 } & \text { CL0029 } & \text { Cupin } \\ \text { PF00190.21 } & \text { CL0029 } & \text { Cupin } \\ \text { PF00190.21 } & \text { CL0029 } & \text { Cupin } \\ \text { PF00190.21 } & \text { CL0029 } & \text { Cupin } \\ \text { PF00190.21 } & \text { CL0029 } & \text { Cupin } \\ \text { PF05899.11 } & \text { CL0029 } & \text { Protein of unknown function (DUF861) } \\ \text { PF00190.21 } & \text { CL0029 } & \text { Cupin } \\ \text { PF00190.21 } & \text { CL0029 } & \text { Cupin } \\ \text { PF00190.21 } & \text { CL0029 } & \text { Cupin } \\ \text { PF00190.21 } & \text { CL0029 } & \text { Cupin } \\ \text { PF00190.21 } & \text { CL0029 } & \text { Cupin } \\ \text { PF00190.21 } & \text { CL0029 } & \text { Cupin } \\ \text { PF13621.5 } & \text { CL0029 } & \text { Cupin-like domain }\end{array}$

1091

\begin{tabular}{clll}
\multicolumn{1}{c}{ Query Name } & \multicolumn{1}{c}{ CDD } & \multicolumn{1}{c}{ SMART } & \multicolumn{1}{c}{ PFAM (HMMER) } \\
Vigun01g148800.1 & monocupin (22-96) & monocupin (22-96) & monocupin (22-96) \\
Vigun01g205600.1 & monocupin (61-207) & monocupin (58-207) & monocupin (60-207) \\
Vigun02g070100.1 & monocupin (61-208) & monocupin (59-208) & monocupin (59-208) \\
Vigun02g110000.1 & monocupin (100-377) & monocupin (100-377) & monocupin (100-377) \\
Vigun02g134000.1 & monocupin (244-393) & monocupin (238-393) & monocupin (238-393) \\
Vigun03g085800.1 & bicupin (12-159) & bicupin (3-159) & bicupin (5-159) \\
& bicupin (35-323) & bicupin (194-240) & bicupin (192-240) \\
Vigun03g085900.1 & monocupin (41-324) & bicupin (3-159) & bicupin (5-159) \\
\hline Vigun03g254300.1 & mo cupin (41-324) & bicupin (192-341) & bicupin (192-341)
\end{tabular}


Vigun03g297500.1

Vigun03g390200.1

Vigun03g397300.1

Vigun03g399100.1

Vigun04g148500.1

Vigun04g167800.1

Vigun04g168100.1

Vigun05g001800.1

Vigun05g152400.1

Vigun05g166300.1

Vigun05g166800.1

Vigun05g166900.1

Vigun05g167000.1

Vigun05g235200.1

Vigun05g250800.1

Vigun05g250900.1

Vigun05g251000.1

Vigun05g254700.1

Vigun06g057200.1

Vigun06g110700.1

Vigun06g138000.1

Vigun06g138100.1

\section{cupin-like(442-605)}

monocupin (52-198)

monocupin (7-165)

monocupin (7-158)

monocupin (21-95)

monocupin (51-125)

pirin (57-310)

Pirin C (57-310)

monocupin (25-55)

monocupin (63-210)

monocupin (63-210)

monocupin (63-210)

monocupin (63-210)

monocupin (52-196)

bicupin (76-192)

bicupin (296-441)

bicupin (74-193)

bicupin (296-441)

bicupin (2-113)

bicupin (216-362)

monocupin (48-354)

No cupin (48-354)

pirin C (24-279)

No cupin domain

LacAB rpiB (11-131)

cupin-like (159-268)

monocupin (59-208)

monocupin (59-209) monocupin (220-289)

JmjC (434-573)

monocupin (52-198)

monocupin (6-166)

monocupin (6-159)

monocupin (21-95)

monocupin (51-125)

Pirin (59-154)

pirin C (207-312)

monocupin (21-58)

monocupin (59-210)

monocupin (59-210)

monocupin (59-210)

monocupin (59-210)

monocupin (52-196)

bicupin (40-193)

bicupin(292-441)

bicupin (58-194)

bicupin(292-441)

bicupin (2-114)

bicupin(213-362)

bicupin (3-157)

bicupin (195-339)

Pirin (30-126)

Pirin C (179-284)

LacAB rpiB (11-139)

bicupin (202-269)

monocupin (59-209)

monocupin (59-209) monocupin (220-289)

cupin-like (439-575)

monocupin (52-197)

monocupin (6-166)

monocupin (6-159)

monocupin (21-95)

monocupin (51-125)

Pririn (59-154)

Pirin C (207-312)

monocupin (21-58)

monocupin (60-210)

monocupin (60-210)

monocupin (60-210)

monocupin (60-210)

monocupin (53-196)

bicupin (43-192)

bicupin(292-441)

bicupin (43-194)

bicupin(292-441)

bicupin (2-113)

bicupin(213-362)

bicupin (6-157)

bicupin (190-339)

Pirin (30-126)

Pirin C (179-284)

LacAB rpiB (11-139)

bicupin (202-269)

monocupin (59-209)

monocupin (59-209) 


\begin{tabular}{|c|c|c|c|}
\hline Vigun07g059500.1 & monocupin (70-212) & monocupin (68-213) & monocupin (67-213) \\
\hline Vigun07g089100.1 & monocupin (22-96) & monocupin (22-96) & monocupin (22-96) \\
\hline \multirow{2}{*}{ Vigun07g100400.1 } & monocupin (25-594) & bicupin (39-234) & bicupin (39-192) \\
\hline & No cupin (25-594) & bicupin (443-589) & bicupin (443-589) \\
\hline Vigun07g106500.1 & monocupin (18-292) & monocupin (146-303) & monocupin (18-2953) \\
\hline Vigun07g108200.1 & monocupin (323-481) & monocupin (320-484) & monocupin (320-480) \\
\hline Vigun07g113800.1 & monocupin (27-192) & monocupin (70-150) & monocupin (70-150) \\
\hline Vigun07g132600.1 & monocupin (62-209) & monocupin (61-209) & monocupin (61-209) \\
\hline Vigun07g132700.1 & monocupin (62-209) & monocupin (61-209) & monocupin (61-209) \\
\hline Vigun07g133900.1 & monocupin (62-208) & monocupin (59-208) & monocupin (60-208) \\
\hline Vigun07g159700.1 & monocupin (26-100) & monocupin (26-100) & monocupin (26-100) \\
\hline Vigun07g160600.1 & monocupin (67-215) & monocupin (67-215) & monocupin (65-215) \\
\hline Vigun07g235300.1 & ARD (4-158)-No cupin & monocupin (83-143) & monocup \\
\hline Vigun07g237100.1 & $\begin{array}{l}\text { bicupin (59-205) } \\
\text { bicupin (261-417) }\end{array}$ & $\begin{array}{l}\text { bicupin }(56-206) \\
\text { bicupin }(258-417)\end{array}$ & $\begin{array}{l}\text { bicupin }(5 \\
\text { bicupin }(2\end{array}$ \\
\hline Vigun07g237200.1 & $\begin{array}{l}\text { bicupin (140-281) } \\
\text { bicupin (344-495) }\end{array}$ & $\begin{array}{l}\text { bicupin }(138-288) \\
\text { bicupin }(341-497)\end{array}$ & $\begin{array}{l}\text { bicupin }(140-288) \\
\text { bicupin }(341-496)\end{array}$ \\
\hline Vigun07g237300.1 & $\begin{array}{l}\text { bicupin (59-205) } \\
\text { bicupin (261-417) }\end{array}$ & $\begin{array}{l}\text { bicupin }(56-206) \\
\text { bicupin }(258-417)\end{array}$ & $\begin{array}{l}\text { bicupin }(56-206) \\
\text { bicupin }(258-417)\end{array}$ \\
\hline Vigun07g237400.1 & $\begin{array}{l}\text { bicupin(57-203) } \\
\text { bicupin (259-415) }\end{array}$ & $\begin{array}{l}\text { bicupin(54-204) } \\
\text { bicupin (256-415) }\end{array}$ & $\begin{array}{l}\text { bicupin(54-204) } \\
\text { bicupin (256-415) }\end{array}$ \\
\hline Vigun07g237500.1 & $\begin{array}{l}\text { bicupin }(57-203) \\
\text { bicupin }(259-415)\end{array}$ & $\begin{array}{l}\text { bicupin }(54-204) \\
\text { bicupin }(256-415)\end{array}$ & $\begin{array}{l}\text { bicupin }(54-204) \\
\text { bicupin }(256-415)\end{array}$ \\
\hline Vigun07g237600.1 & $\begin{array}{l}\text { bicupin(196-346) } \\
\text { bicupin(398-551) }\end{array}$ & $\begin{array}{l}\text { bicupin (188-347) } \\
\text { bicupin 395-341 }\end{array}$ & $\begin{array}{l}\text { no domain cupin } \\
\text { monocupin (395-551) }\end{array}$ \\
\hline Vigun08g174800.1 & monocupin (61-135) & monocupin (61-135) & monocupin (61-135) \\
\hline \multirow{2}{*}{ Vigun08g205900.1 } & Pirin C (14-279) & Pirin (61-135) & Pirin (30-126) \\
\hline & No domain & Piri & Pirin C (179- \\
\hline
\end{tabular}




\begin{tabular}{|c|c|c|c|}
\hline \multirow{2}{*}{ Vigun09g089200.1 } & cupin like (20-289) & JmjC (122-302) & Cupin_8 (19-291) \\
\hline & No domain & No domain & JmjC (179-285) \\
\hline Vigun09g122200.1 & monocupin (78-181) & monocupin (66-208) & monocupin (66-202) \\
\hline \multirow{2}{*}{ Vigun09g177900.1 } & F-box-like (15-59) & F-box-like (18-58) & F-box-like (18-58) \\
\hline & cupin-like (135-367) & JmjC (210-337) & cupin_8 (135-367) \\
\hline \multirow{2}{*}{ Vigun10g081000.1 } & bicupin (57-203) & bicupin (46-204) & bicupin (51-204) \\
\hline & bicupin (257-417) & bicupin (254-417) & bicupin (254-417) \\
\hline Vigun10g086400.1 & monocupin (62-209) & monocupin (60-209) & monocupin (60-209) \\
\hline Vigun10g086500.1 & monocupin (62-209) & monocupin (60-209) & monocupin (60-209) \\
\hline Vigun10g086600.1 & monucupin (64-209) & monocupin (61-209) & monocupin (62-209) \\
\hline Vigun10g086700.1 & monocupin (62-209) & monocupin (60-209) & monocupin (60-209) \\
\hline Vigun10g087300.1 & monocupin (62-209) & monocupin (60-209) & monocupin (60-209) \\
\hline \multirow{2}{*}{ Vigun10g096400.1 } & bicupin (57-203) & bicupin (54-204) & bicupin (54-204) \\
\hline & bicupin (259-423) & bicupin (256-423) & bicupin (256-423) \\
\hline \multirow{2}{*}{ Vigun10g096600.1 } & bicupin (259-423) & bicupin (54-204) & bicupin (54-204) \\
\hline & bicupin (259-423) & bicupin (256-423) & bicupin (256-423) \\
\hline \multirow{2}{*}{ Vigun10g101100.1 } & F-box-like (100-139) & F-box-like (98-138) & JmjC (323-434) \\
\hline & cupin-like (215-434) & JmjC (282-451) & Cupin_8 (2014-439) \\
\hline Vigun10g164200.1 & monocupin (66-199) & monocupin (55-199) & monocupin (57-199 \\
\hline Vigun10g164300.1 & monocupin (60-199) & monocupin (55-199) & monocupin (54-199) \\
\hline Vigun10g164400.1 & monocupin (54-196) & monocupin (52-196) & monocupin (51-196) \\
\hline Vigun10g164500.1 & monocupin (69-202) & monocupin (58-202) & monocupin (60-202) \\
\hline Vigun10g164600.1 & monocupin (60-199) & monocupin (55-199) & monocupin (54-199) \\
\hline Vigun10g164700.1 & monocupin (54-196) & monocupin (52-196) & monocupin (52-196) \\
\hline Vigun10g164800.1 & monocupin (54-199) & monocupin (53-199) & monocupin (53-199) \\
\hline \multirow{2}{*}{ Vigun11g024500.1 } & bicupin (54-200) & bicupin (51-201) & bicupin (44-201) \\
\hline & bicupin (252-410) & bicupin (249-411) & bicupin (249-410) \\
\hline Vigun11g026700.1 & monocupin (78-188) & monocupin (66-201) & monocupin (67-2011) \\
\hline Vigun11g038900.1 & monocupin (173-203) & monocupin (169-206) & monocupin (169-206) \\
\hline
\end{tabular}




\begin{tabular}{|llll} 
Vigun11g098100.1 & bicupin (55-201) & bicupin (52-202) & bicupin (51-202) \\
& bicupin (256-408) & bicupin (252-409) & bicupin (252-409) \\
Vigun11g147100.1 & bicupin (35-187) & bicupin (28-187) & bicupin (31-146) \\
& bicupin (278-424) & bicupin (275-424) & bicupin (275-424) \\
Vigun11g151800.1 & No cupin (48-354) & bicupin (3-157) & bicupin (5-157) \\
& No cupin (48-354) & bicupin (195-339) & bicupin (190-339)
\end{tabular}

1093 Vigna algulares

\begin{tabular}{|c|c|c|c|c|c|}
\hline Query Name & Hits Found & Identifier & Accession & Clan & Description \\
\hline ВАТ97405.1 & & Cupin_1 & PF00190.21 & CL0029 & Cupin \\
\hline КОМ36077.1 & & Cupin_1 & PF00190.21 & CL0029 & Cupin \\
\hline КОМ36078.1 & & Cupin_1 & PF00190.21 & CL0029 & Cupin \\
\hline XP_017411733.1 & & Cupin_1 & PF00190.21 & CL0029 & Cupin \\
\hline XP_017413272.1 & & Cupin_1 & PF00190.21 & CL0029 & Cupin \\
\hline XP_017413273.1 & & Cupin_1 & PF00190.21 & CL0029 & Cupin \\
\hline XP_017413275.1 & & Cupin_1 & PF00190.21 & CL0029 & Cupin \\
\hline XP_017414388.1 & & Cupin_1 & PF00190.21 & CL0029 & Cupin \\
\hline
\end{tabular}

\begin{tabular}{|c|c|c|c|}
\multicolumn{1}{|c}{ Start } & \multicolumn{2}{c}{ End } & \multicolumn{2}{c}{$\begin{array}{c}\text { Domain E-values } \\
\text { Individual }\end{array}$} & \multicolumn{1}{c|}{ Conditional } \\
\hline 51 & 204 & $1,30 \mathrm{E}-07$ & $7,60 \mathrm{E}-12$ \\
\hline 256 & 416 & $3,30 \mathrm{E}-27$ & $2,00 \mathrm{E}-31$ \\
\hline 56 & 207 & $2,20 \mathrm{E}-06$ & $1,30 \mathrm{E}-10$ \\
\hline 259 & 378 & $8,00 \mathrm{E}-13$ & $4,80 \mathrm{E}-17$ \\
\hline 120 & 269 & 0,00026 & $1,50 \mathrm{E}-08$ \\
\hline 321 & 476 & $1,90 \mathrm{E}-24$ & $1,10 \mathrm{E}-28$ \\
\hline 79 & 194 & $1,10 \mathrm{E}-12$ & $1,30 \mathrm{E}-16$ \\
\hline 322 & 471 & $1,00 \mathrm{E}-25$ & $1,20 \mathrm{E}-29$ \\
\hline 57 & 207 & $1,20 \mathrm{E}-07$ & $7,10 \mathrm{E}-12$ \\
\hline 259 & 417 & $1,70 \mathrm{E}-25$ & $1,00 \mathrm{E}-29$ \\
\hline 56 & 207 & $3,40 \mathrm{E}-08$ & $2,00 \mathrm{E}-12$ \\
\hline 259 & 421 & $8,70 \mathrm{E}-27$ & $5,20 \mathrm{E}-31$ \\
\hline 193 & 347 & $3,40 \mathrm{E}-05$ & $2,00 \mathrm{E}-09$ \\
\hline 397 & 553 & $2,50 \mathrm{E}-25$ & $1,50 \mathrm{E}-29$ \\
\hline 120 & 269 & 0,00018 & $1,10 \mathrm{E}-08$ \\
\hline
\end{tabular}




\begin{tabular}{|c|c|c|c|c|c|c|c|c|c|}
\hline \multirow{3}{*}{ XP_017414389.1 } & & \multirow{3}{*}{ Cupin_1 } & \multirow{3}{*}{ PF00190.21 } & \multirow{3}{*}{ CL0029 } & \multirow{3}{*}{ Cupin } & 321 & 476 & $1,30 E-24$ & 7,70 E-29 \\
\hline & & & & & & 56 & 207 & 3,00E-06 & $1,80 \mathrm{E}-10$ \\
\hline & & & & & & 259 & 420 & $1,30 E-26$ & $7,60 E-31$ \\
\hline XP_017415489.1 & & Cupin_1 & PF00190.21 & CL0029 & Cupin & 320 & 480 & $2,10 \mathrm{E}-27$ & 1,30E-31 \\
\hline \multirow{10}{*}{ XP_017433627.1 } & \multirow{10}{*}{ Hits Found } & \multirow{10}{*}{ Cupin_1 } & \multirow{10}{*}{ PF00190.21 } & \multirow{10}{*}{ CL0029 } & \multirow{10}{*}{ Cupin } & 49 & 205 & 1,40E-08 & $8,20 E-13$ \\
\hline & & & & & & 257 & 420 & $2,80 E-26$ & $1,70 E-30$ \\
\hline & & & & & & Start & End & Individual & Conditional \\
\hline & & & & & & 10 & 131 & $6,30 \mathrm{E}-16$ & $3,80 \mathrm{E}-20$ \\
\hline & & & & & & 5 & 159 & $4,10 \mathrm{E}-20$ & $4,90 \mathrm{E}-24$ \\
\hline & & & & & & 192 & 341 & $5,20 \mathrm{E}-18$ & $6,30 E-22$ \\
\hline & & & & & & 39 & 192 & $1,20 \mathrm{E}-25$ & $7,50 \mathrm{E}-30$ \\
\hline & & & & & & 450 & 596 & $6,30 E-30$ & $3,80 \mathrm{E}-34$ \\
\hline & & & & & & 5 & 157 & $5,60 \mathrm{E}-28$ & $3,30 \mathrm{E}-32$ \\
\hline & & & & & & 190 & 339 & $9,40 \mathrm{E}-23$ & $5,60 \mathrm{E}-27$ \\
\hline
\end{tabular}

1094 Vigna rariata

\begin{tabular}{|c|c|c|c|c|c|c|c|c|}
\hline \multirow{3}{*}{ NP_001304202 } & \multirow{3}{*}{ Cupin_1 } & \multirow{3}{*}{ PF00190.21 } & \multirow{3}{*}{ CL0029 } & \multirow{3}{*}{ Cupin } & & & & \\
\hline & & & & & 56 & 206 & 8,00E-08 & $4,80 \mathrm{E}-12$ \\
\hline & & & & & 259 & 420 & 1,70E-26 & $1,00 \mathrm{E}-30$ \\
\hline \multirow{2}{*}{ NP_001304231.1 } & \multirow{2}{*}{ Cupin_1 } & \multirow{2}{*}{ PF00190.21 } & \multirow{2}{*}{ CL0029 } & \multirow{2}{*}{ Cupin } & 47 & 204 & $3,00 E-08$ & $1,80 \mathrm{E}-12$ \\
\hline & & & & & 256 & 413 & $8,90 \mathrm{E}-29$ & $5,30 \mathrm{E}-33$ \\
\hline \multirow{2}{*}{ XP_014492536.1 } & \multirow{2}{*}{ Cupin_1 } & \multirow{2}{*}{ PF00190.21 } & \multirow{2}{*}{ CL0029 } & \multirow{2}{*}{ Cupin } & 49 & 205 & $3,10 \mathrm{E}-08$ & $3,70 \mathrm{E}-12$ \\
\hline & & & & & 257 & 413 & $3,50 \mathrm{E}-26$ & $4,10 \mathrm{E}-30$ \\
\hline \multirow{2}{*}{ XP_014493578.1 } & \multirow{2}{*}{ Cupin_1 } & \multirow{2}{*}{ PF00190.21 } & \multirow{2}{*}{ CL0029 } & \multirow{2}{*}{ Cupin } & 83 & 196 & $1,10 \mathrm{E}-10$ & $1,30 \mathrm{E}-14$ \\
\hline & & & & & 323 & 472 & $5,30 \mathrm{E}-25$ & $6,30 \mathrm{E}-29$ \\
\hline XP_014512682.1 & Cupin_1 & PF00190.21 & CL0029 & Cupin & 318 & 479 & $1,80 \mathrm{E}-28$ & $1,10 \mathrm{E}-32$ \\
\hline XP_014507363.1 & Cupin_1 & PF00190.21 & CL0029 & Cupin & 58 & 206 & $6,20 \mathrm{E}-08$ & $3,70 \mathrm{E}-12$ \\
\hline
\end{tabular}




\section{XP_014523923.1}

XP_014523928.1

XP_014523936.1

XP_014523938.1

XP_014524354.1

1095

Vigna radiata legumins

\section{XP_014506003.1}

XP_014508262.1

XP_014508263.1

XP_014521758.1

1096
Cupin_1 PF00190.21 CL0029 Cupin

Cupin_1 PF00190.21 CL0029 Cupin

Cupin_1 PF00190.21 CL0029 Cupin

Cupin_1 PF00190.21 CL0029 Cupin

Cupin_1 PF00190.21 CL0029 Cupin

\begin{tabular}{|c|c|c|c|}
\hline 259 & 413 & $1,70 \mathrm{E}-29$ & $9,90 \mathrm{E}-34$ \\
\hline 57 & 206 & $4,20 \mathrm{E}-08$ & $2,50 \mathrm{E}-12$ \\
\hline 206 & 339 & $7,10 \mathrm{E}-18$ & $4,20 \mathrm{E}-22$ \\
\hline 200 & 353 & $5,50 \mathrm{E}-05$ & $3,30 \mathrm{E}-09$ \\
\hline 403 & 560 & $2,60 \mathrm{E}-25$ & $1,60 \mathrm{E}-29$ \\
\hline 129 & 278 & $7,60 \mathrm{E}-05$ & $4,50 \mathrm{E}-09$ \\
\hline 329 & 485 & $2,30 \mathrm{E}-25$ & $1,40 \mathrm{E}-29$ \\
\hline 58 & 206 & $6,90 \mathrm{E}-08$ & $4,10 \mathrm{E}-12$ \\
\hline 259 & 420 & $9,70 \mathrm{E}-27$ & $5,80 \mathrm{E}-31$ \\
\hline 57 & 206 & $1,30 \mathrm{E}-08$ & $8,10 \mathrm{E}-13$ \\
\hline 259 & 419 & $8,90 \mathrm{E}-27$ & $5,40 \mathrm{E}-31$ \\
\hline
\end{tabular}

$\begin{array}{llll}\text { Cupin_1 } & \text { PF00190.21 } & \text { CL0029 } & \text { Cupin } \\ \text { Cupin_1 } & \text { PF00190.21 } & \text { CL0029 } & \text { Cupin } \\ & & & \\ \text { Cupin_1 } & \text { PF00190.21 } & \text { CL0029 } & \text { Cupin }\end{array}$

\begin{tabular}{|c|c|c|c|}
\hline 25 & 179 & $9,20 \mathrm{E}-22$ & $1,10 \mathrm{E}-25$ \\
\hline 212 & 361 & $1,60 \mathrm{E}-15$ & $1,90 \mathrm{E}-19$ \\
\hline 5 & 159 & $4,60 \mathrm{E}-21$ & $5,50 \mathrm{E}-25$ \\
\hline 192 & 321 & $6,80 \mathrm{E}-14$ & $8,10 \mathrm{E}-18$ \\
\hline 5 & 159 & $4,30 \mathrm{E}-21$ & $5,10 \mathrm{E}-25$ \\
\hline 192 & 314 & $6,50 \mathrm{E}-14$ & $7,70 \mathrm{E}-18$ \\
\hline 39 & 192 & $9,40 \mathrm{E}-26$ & $1,10 \mathrm{E}-29$ \\
\hline 445 & 591 & $3,10 \mathrm{E}-31$ & $3,70 \mathrm{E}-35$ \\
\hline
\end{tabular}

Phvul.002G239800

Cupin_1 smart00835

Cupin; This family represents the conserved barrel domain of the 'cupin' superfamily ('cupa' ... Phvul.002G239900 Phvul.002G239900 Phvul.002G249800 No cupin cl28274

cl28274 glutelin; Provisional, No vicilin domain glutelin; Provisional, No vicilin domain

Cupin_1

Cupin; This family represents the conserved barrel domain of the 'cupin' superfamily ('cupa' ... 


\begin{tabular}{|c|c|c|c|}
\hline Phvul.003G131400 & Cupin_1 & pfam00190 & Cupin; This family represents the conserved barrel domain of the 'cupin' superfamily ('cupa' ... \\
\hline \multirow{2}{*}{ Phvul.005G137200 } & Cupin_1 & smart00835 & Cupin; This family represents the conserved barrel domain of the 'cupin' superfamily ('cupa' \\
\hline & Cupin_1 & smart00835 & Cupin; This family represents the conserved barrel domain of the 'cupin' superfamily ('cupa' \\
\hline Phvul.007G173600 & $\begin{array}{l}\text { Cupin_1 } \\
\text { cupin like super family }\end{array}$ & $\begin{array}{l}\text { smart00835 } \\
\text { cl21464 }\end{array}$ & $\begin{array}{l}\text { Cupin; This family represents the conserved barrel domain of the 'cupin' superfamily ('cupa' ... } \\
\text { onserved domain found in cupin and related proteins; A diverse protein domains superfamily .. }\end{array}$ \\
\hline Phvul.007G192800 & No cupin & cl28274 & glutelin; Provisional, No vicilin domain \\
\hline Phvul.007G206600 & Cupin_1 & pfam00190 & Cupin; This family represents the conserved barrel domain of the 'cupin' superfamily ('cupa' ... \\
\hline Phvul.007G207800 & Cupin_1 & pfam00190 & Cupin; This family represents the conserved barrel domain of the 'cupin' superfamily ('cupa' ... \\
\hline Phvul.007G229500 & Cupin_1 & pfam00190 & Cupin; This family represents the conserved barrel domain of the 'cupin' superfamily ('cupa' ... \\
\hline Phvul.009G227800 & Cupin_1 & pfam00190 & Cupin; This family represents the conserved barrel domain of the 'cupin' superfamily ('cupa' ... \\
\hline Phvul.010G129800 & Cupin_1 & pfam00190 & Cupin; This family represents the conserved barrel domain of the 'cupin' superfamily ('cupa' ... \\
\hline Phvul.011G067800 & No cupin & $\mathrm{cl} 28274$ & glutelin; Provisional, No vicilin domain \\
\hline \multirow{2}{*}{ Phvul.011G072800 } & Cupin_1 & smart00835 & Cupin; This family represents the conserved barrel domain of the 'cupin' superfamily ('cupa' \\
\hline & Cupin_1 & smart00835 & Cupin; This family represents the conserved barrel domain of the 'cupin' superfamily ('cupa' \\
\hline Phvul.L001743 & No cupin & cl28274 & glutelin; Provisional, No vicilin domain \\
\hline
\end{tabular}

\begin{tabular}{|c|c|c|c|c|c|c|c|c|}
\hline Phvul.003G131400 & Cupin_1 & PF00190.21 & CL0029 & Cupin & 52 & 197 & $2,20 E-33$ & $2,60 \mathrm{E}-37$ \\
\hline \multirow[t]{2}{*}{ Phvul.005G137200 } & Cupin_1 & PF00190.21 & CL0029 & Cupin & 43 & 198 & $5,40 \mathrm{E}-09$ & $6,40 \mathrm{E}-13$ \\
\hline & & & & & 297 & 446 & $1,40 E-32$ & $1,60 \mathrm{E}-36$ \\
\hline \multirow[t]{2}{*}{ Phvul.007G173600 } & Cupin_1 & PF00190.21 & CL0029 & Cupin & 121 & 260 & $7,00 \mathrm{E}-05$ & $4,20 \mathrm{E}-09$ \\
\hline & & & & & 314 & 475 & $1,60 \mathrm{E}-29$ & $9,80 \mathrm{E}-34$ \\
\hline \multirow[t]{2}{*}{ Phvul.007G192800.1 } & Cupin_1 & PF00190.21 & CL0029 & Cupin & 36 & 188 & $3,70 \mathrm{E}-26$ & $2,20 \mathrm{E}-30$ \\
\hline & & & & & 458 & 604 & $9,80 E-32$ & $5,90 \mathrm{E}-36$ \\
\hline Phvul.007G206600 & Cupin_1 & PF00190.21 & CL0029 & Cupin & 65 & 213 & $7,70 \mathrm{E}-48$ & $9,30 \mathrm{E}-52$ \\
\hline Phvul.007G207800 & Cupin_1 & PF00190.21 & CL0029 & Cupin & 61 & 209 & $2,30 \mathrm{E}-48$ & $2,70 E-52$ \\
\hline Phvul.007G229500 & Cupin_1 & PF00190.21 & CL0029 & Cupin & 67 & 213 & $7,40 \mathrm{E}-43$ & $8,90 \mathrm{E}-47$ \\
\hline Phvul.009G227800 & Cupin_1 & PF00190.21 & CL0029 & Cupin & 54 & 200 & $5,80 \mathrm{E}-35$ & $7,00 E-39$ \\
\hline Phvul.010G129800 & Cupin_1 & PF00190.21 & CL0029 & Cupin & 53 & 198 & $1,10 \mathrm{E}-29$ & $1,40 E-33$ \\
\hline
\end{tabular}




\begin{tabular}{|c|c|c|c|c|c|c|c|c|}
\hline \multirow[t]{2}{*}{ Phvul.011G067800.1 } & \multirow{2}{*}{ Cupin_1 } & \multirow{2}{*}{ PF00190.21 } & \multirow{2}{*}{ CL0029 } & \multirow{2}{*}{ Cupin } & 5 & 157 & $7,80 \mathrm{E}-29$ & $4,70 E-33$ \\
\hline & & & & & 190 & 339 & $9,00 E-22$ & $5,40 E-26$ \\
\hline \multirow[t]{2}{*}{ Phvul.011G072800.2 } & \multirow[t]{2}{*}{ Cupin_1 } & \multirow[t]{2}{*}{ PF00190.21 } & \multirow[t]{2}{*}{ CL0029 } & \multirow[t]{2}{*}{ Cupin } & 32 & 148 & $2,10 \mathrm{E}-10$ & $2,50 \mathrm{E}-14$ \\
\hline & & & & & 273 & 422 & $5,40 \mathrm{E}-31$ & $6,50 \mathrm{E}-35$ \\
\hline \multirow[t]{2}{*}{ Phvul.L001743 } & \multirow[t]{2}{*}{ Cupin_1 } & \multirow[t]{2}{*}{ PF00190.21 } & \multirow[t]{2}{*}{ CL0029 } & \multirow[t]{2}{*}{ Cupin } & 5 & 157 & $4,30 \mathrm{E}-29$ & $2,60 \mathrm{E}-33$ \\
\hline & & & & & 190 & 339 & $6,10 \mathrm{E}-22$ & $3,70 E-26$ \\
\hline Phvul.003G131400 & Cupin_1 & PF00190.21 & CL0029 & Cupin & 52 & 197 & $2,20 \mathrm{E}-33$ & $2,60 E-37$ \\
\hline \multirow{2}{*}{ Phvul.005G137200 } & \multirow{2}{*}{ Cupin_1 } & \multirow{2}{*}{ PF00190.21 } & \multirow{2}{*}{ CL0029 } & \multirow{2}{*}{ Cupin } & 43 & 198 & $5,40 \mathrm{E}-09$ & $6,40 \mathrm{E}-13$ \\
\hline & & & & & 297 & 446 & $1,40 \mathrm{E}-32$ & $1,60 \mathrm{E}-36$ \\
\hline \multirow{2}{*}{ Phvul.007G173600 } & \multirow{2}{*}{ Cupin_1 } & \multirow{2}{*}{ PF00190.21 } & \multirow{2}{*}{ CL0029 } & \multirow{2}{*}{ Cupin } & 121 & 260 & $7,00 \mathrm{E}-05$ & $4,20 E-09$ \\
\hline & & & & & 314 & 475 & $1,60 \mathrm{E}-29$ & $9,80 \mathrm{E}-34$ \\
\hline \multirow{2}{*}{ Phvul.007G192800.1 } & \multirow{2}{*}{ Cupin_1 } & \multirow{2}{*}{ PF00190.21 } & \multirow[b]{2}{*}{ CL0029 } & \multirow[b]{2}{*}{ Cupin } & 36 & 188 & $3,70 \mathrm{E}-26$ & $2,20 \mathrm{E}-30$ \\
\hline & & & & & 458 & 604 & $9,80 \mathrm{E}-32$ & $5,90 \mathrm{E}-36$ \\
\hline Phvul.007G206600 & Cupin_1 & PF00190.21 & CL0029 & Cupin & 65 & 213 & $7,70 E-48$ & $9,30 \mathrm{E}-52$ \\
\hline Phvul.007G207800 & Cupin_1 & PF00190.21 & CL0029 & Cupin & 61 & 209 & $2,30 E-48$ & $2,70 \mathrm{E}-52$ \\
\hline Phvul.007G229500 & Cupin_1 & PF00190.21 & CL0029 & Cupin & 67 & 213 & $7,40 \mathrm{E}-43$ & $8,90 \mathrm{E}-47$ \\
\hline Phvul.009G227800 & Cupin_1 & PF00190.21 & CL0029 & Cupin & 54 & 200 & $5,80 \mathrm{E}-35$ & $7,00 \mathrm{E}-39$ \\
\hline Phvul.010G129800 & Cupin_1 & PF00190.21 & CL0029 & Cupin & 53 & 198 & $1,10 E-29$ & $1,40 E-33$ \\
\hline \multirow{2}{*}{ Phvul.011G067800.1 } & \multirow{2}{*}{ Cupin_1 } & \multirow{2}{*}{ PF00190.21 } & Cl0029 & Cuni & 5 & 157 & $7,80 \mathrm{E}-29$ & $4,70 \mathrm{E}-33$ \\
\hline & & & $C L O 0<9$ & Cupin & 190 & 339 & $9,00 \mathrm{E}-22$ & $5,40 E-26$ \\
\hline Phvul 011G0728002 & & PF0019021 & Cl0029 & & 32 & 148 & $2,10 \mathrm{E}-10$ & $2,50 \mathrm{E}-14$ \\
\hline Phvul.011G0/2800.2 & cupin_1 & PFOO190.21 & CLOU 29 & Cupın & 273 & 422 & $5,40 E-31$ & $6,50 E-35$ \\
\hline Phvul.L001743 & Cunin & PF0019021 & Cl0029 & & 5 & 157 & $4,30 E-29$ & $2,60 \mathrm{E}-33$ \\
\hline PrIvui.LU01/43 & Cupin_1 & PF00190.21 & CLOU 29 & Cupl & 190 & 339 & $6,10 \mathrm{E}-22$ & $3,70 \mathrm{E}-26$ \\
\hline
\end{tabular}

1098

$\begin{array}{cccccc}\text { Gene } & \text { Signal peptide } & \text { Name } & \text { Start } & \text { End } & \text { E-value } \\ \text { Phvul.001G221200.1 } & 1-22 & \text { Cupin_1 } & 59 & 208 & 1.9 e-34 \\ \text { Phvul.002G027900.1 } & 1-17 & \text { Cupin_1 } & 233 & 388 & 5,85 E-44\end{array}$




\begin{tabular}{|c|c|c|c|c|c|}
\hline Phvul.002G027900.2 & N/A & Cupin_1 & 90 & 245 & $5,85 E-44$ \\
\hline Phvul.002G077300 & $1-21$ & Cupin_1 & 59 & 208 & $8,29 E-35$ \\
\hline \multirow{2}{*}{ Phvul.002G239800 } & N/A & Pfam:Cupin_1 & 1 & 87 & 0,0000019 \\
\hline & N/A & Cupin_1 & 120 & 228 & 0,00812 \\
\hline \multirow{2}{*}{ Phvul.002G239900.1 } & N/A & Cupin_1 & 3 & 159 & $2,11 \mathrm{E}-27$ \\
\hline & & Cupin_1 & 192 & 341 & $2,10 \mathrm{E}-13$ \\
\hline \multirow{2}{*}{ Phvul.002G239900.2 } & N/A & Pfam:Cupin_1 & 1 & 87 & 0,0000065 \\
\hline & & Cupin_1 & 120 & 269 & $2,10 \mathrm{E}-13$ \\
\hline \multirow{2}{*}{ Phvul.002G249800 } & N/A & Cupin_1 & 3 & 159 & $7,75 E-26$ \\
\hline & & Cupin_1 & 192 & 341 & $1,68 \mathrm{E}-12$ \\
\hline Phvul.003G131400 & $1-17$ & Cupin_1 & 52 & 198 & $1,62 \mathrm{E}-37$ \\
\hline \multirow{2}{*}{ Phvul.005G137200 } & $1-23$ & Cupin_1 & 55 & 198 & $5,09 \mathrm{E}-12$ \\
\hline & & Cupin_1 & 297 & 446 & $5,21 \mathrm{E}-50$ \\
\hline \multirow{2}{*}{ Phvul.007G173600.1 } & $1-24$ & Cupin_1 & 115 & 274 & 0,431 \\
\hline & & Cupin_1 & 314 & 478 & $6,27 E-44$ \\
\hline \multirow{2}{*}{ Phvul.007G192800.1 } & $1-23$ & Cupin_1 & 36 & 228 & $3,93 \mathrm{E}-27$ \\
\hline & & Cupin_1 & 458 & 604 & $9,08 E-41$ \\
\hline Phvul.007G206600.1 & $1-27$ & Cupin_1 & 64 & 213 & $8,42 E-36$ \\
\hline Phvul.007G207800.1 & $1-22$ & Cupin_1 & 61 & 209 & $5,73 E-38$ \\
\hline Phvul.007G229500 & $1-23$ & Cupin_1 & 67 & 213 & $5,94 \mathrm{E}-45$ \\
\hline Phvul.009G227800 & $1-19$ & Cupin_1 & 54 & 200 & $8,60 \mathrm{E}-42$ \\
\hline Phvul.010G129800 & $1-19$ & Cupin_1 & 54 & 198 & $1,24 \mathrm{E}-33$ \\
\hline \multirow{2}{*}{ Phvul.011G067800 } & N/A & Cupin_1 & 3 & 157 & $5,07 E-30$ \\
\hline & & Cupin_1 & 195 & 339 & $3,23 \mathrm{E}-14$ \\
\hline \multirow{2}{*}{ Phvul.011G072800 } & $1-21$ & Cupin_1 & 31 & 187 & $1,04 \mathrm{E}-17$ \\
\hline & & Cupin_1 & 237 & 422 & $1,65 E-52$ \\
\hline \multirow{2}{*}{ Phvul.L001743 } & N/A & Cupin_1 & 3 & 157 & $6,10 \mathrm{E}-33$ \\
\hline & & Cupin_1 & 195 & 339 & $6,21 E-16$ \\
\hline
\end{tabular}

